\title{
Endoplasmic reticulum-associated degradation is required for nephrin maturation and kidney glomerular filtration function
}

\author{
Sei Yoshida, ${ }^{1,2}$ Xiaoqiong Wei, ${ }^{1}$ Gensheng Zhang, ${ }^{1}$ Christopher L. O’Connor, ${ }^{3}$ Mauricio Torres, ${ }^{1}$ Zhangsen Zhou, ${ }^{1}$ Liangguang Lin, ${ }^{1}$ \\ Rajasree Menon, ${ }^{3}$ Xiaoxi Xu, ${ }^{4}$ Wenyue Zheng, ${ }^{2}$ Yi Xiong, ${ }^{5}$ Edgar Otto, ${ }^{3}$ Chih-Hang Anthony Tang, ${ }^{6}$ Rui Hua, ${ }^{2}$ Rakesh Verma, ${ }^{3}$ \\ Hiroyuki Mori, ${ }^{1}$ Yang Zhang, ${ }^{7}$ Chih-Chi Andrew Hu, ${ }^{6}$ Ming Liu, ${ }^{4}$ Puneet Garg, ${ }^{3}$ Jeffrey B. Hodgin, ${ }^{8}$ Shengyi Sun, ${ }^{5}$ \\ Markus Bitzer, ${ }^{3}$ and Ling Q $\mathbf{i}^{1,9}$ \\ 'Department of Molecular and Integrative Physiology, University of Michigan Medical School, Ann Arbor, Michigan, USA. 25tate Key Laboratory of Medical Chemical Biology, College of Life Sciences, Frontiers \\ Science Center for Cell Responses, Nankai University, Tianjin, China. ${ }^{3}$ Division of Nephrology, Department of Internal Medicine, University of Michigan Medical School, Ann Arbor, Michigan, USA. ${ }^{4}$ Department \\ of Endocrinology and Metabolism, Tianjin Medical University General Hospital, Tianjin, China. ${ }^{5}$ Center for Molecular Medicine and Cenetics, Department of Biochemistry, Microbiology and Immunology, \\ Wayne State University School of Medicine, Detroit, Michigan, USA. ${ }^{6}$ Houston Methodist Cancer Center, Houston Methodist Academic Institute, Houston, Texas, USA. ${ }^{7}$ Department of Computational Medicine \\ and Bioinformatics and Department of Biological Chemistry and ${ }^{8}$ Department of Pathology and ${ }^{9}$ Division of Metabolism, Endocrinology \& Diabetes, Department of Internal Medicine, University of Michigan \\ Medical School, Ann Arbor, Michigan, USA.
}

\begin{abstract}
Podocytes are key to the glomerular filtration barrier by forming a slit diaphragm between interdigitating foot processes; however, the molecular details and functional importance of protein folding and degradation in the ER remain unknown. Here, we show that the SEL1L-HRD1 protein complex of ER-associated degradation (ERAD) is required for slit diaphragm formation and glomerular filtration function. SEL1L-HRD1 ERAD is highly expressed in podocytes of both mouse and human kidneys. Mice with podocyte-specific Sel1L deficiency develop podocytopathy and severe congenital nephrotic syndrome with an impaired slit diaphragm shortly after weaning and die prematurely, with a median lifespan of approximately 3 months. We show mechanistically that nephrin, a type 1 membrane protein causally linked to congenital nephrotic syndrome, is an endogenous ERAD substrate. ERAD deficiency attenuated the maturation of nascent nephrin, leading to its retention in the ER. We also show that various autosomal-recessive nephrin disease mutants were highly unstable and broken down by SEL1L-HRD1 ERAD, which attenuated the pathogenicity of the mutants toward the WT allele. This study uncovers a critical role of SEL1L-HRD1 ERAD in glomerular filtration barrier function and provides insights into the pathogenesis associated with autosomal-recessive disease mutants.
\end{abstract}

\section{Introduction}

The key function of the kidneys is the ultrafiltration of blood in the glomerulus, where podocytes, specialized differentiated epithelial cells, wrap around capillaries of the glomerulus via a unique cellular structure called foot process (FP) (1). The interaction between interdigitating FPs is connected by a specialized cell junction known as the slit diaphragm, forming the filtration barrier. The slit diaphragm is composed of multiprotein complexes including the membrane proteins nephrin and podocin, as well as cytosolic adapter protein $\mathrm{CD} 2$-associated protein (CD2AP), adhesion protein zonula occludens 1 (ZO1), and actin-associated protein synaptopodin (2-4). Loss of function of any of these factors in mice and humans leads to podocyte injury (i.e., podocytopathy) and renal failure due to impaired FP function and disruption of the slit diaphragm (3-8).

Authorship note: SY, XW, and CZ are co-first authors.

Conflict of interest: The authors have declared that no conflict of interest exists.

Copyright: (ㄷ 2021, American Society for Clinical Investigation.

Submitted: September 4, 2020; Accepted: February 11, 2021; Published: April 1, 2021.

Reference information: J Clin Invest. 2021;131(7):e143988.

https://doi.org/10.1172/JCl143988.
Congenital nephrotic syndrome of the Finnish type (NPHS1, MIM \#256300) is an autosomal-recessive disease in which the nephrin gene is mutated $(3,7)$, and is characterized by massive proteinuria, lack of slit diaphragms and FPs, and premature death before 3 months of age in humans if left untreated (9-12). In mice, loss of nephrin leads to death within 24 hours of birth (8, 13). Nephrin is a type 1 transmembrane protein belonging to the Ig superfamily, with 10 glycosylation sites and 8 disulfide bonds (14). However, molecular events underlying the biogenesis of nascent nephrin proteins as well as the role and significance of ER quality control machineries in this process remain largely unexplored. Several of the over 250 nephrin mutations, mostly autosomal-recessive in humans, have been shown to be retained in the ER, pointing to the significance of these questions $(14,15)$.

ER homeostasis has been proposed to be important for podocyte function and the development of kidney diseases, although the precise mechanism remains unknown (16-18). ER homeostasis is primarily maintained by 3 principal quality control machineries, namely ER-associated degradation (ERAD), the unfolded protein response (UPR), and macroautophagy (hereafter referred to as autophagy). Activation of the mTORC1 pathway evokes ER stress, 
A

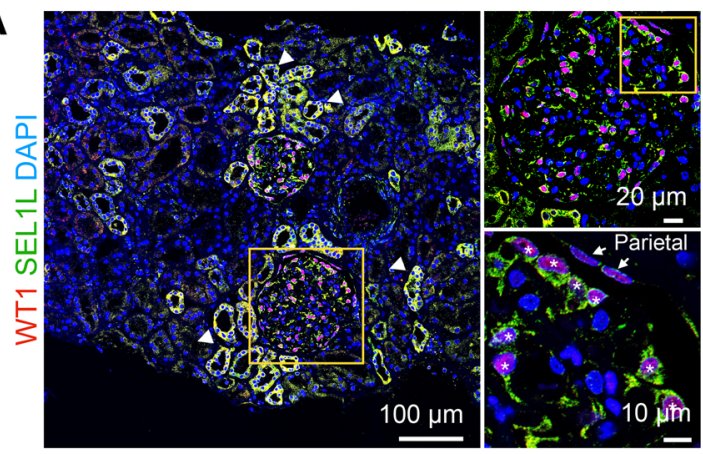

B

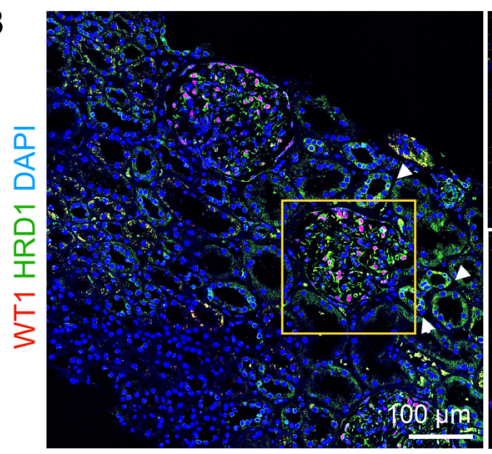

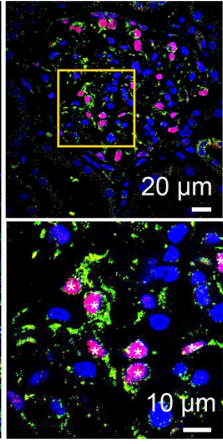

Figure 1. SEL1L-HRD1 ERAD is expressed in human podocytes. Representative confocal images of SEL1L (A) and HRD1 (B) costaining with WT1 in kidney tissues from healthy humans. Asterisks identify WT1+ podocytes. Arrowheads indicate distal tubular cells also expressing SEL1L and HRD1. In addition to expression in podocytes, WT1 was also expressed in parietal epithelial cells lining the Bowman capsule (arrows). Scale bars: $100 \mu \mathrm{m}, 20 \mu \mathrm{m}$, and $10 \mu \mathrm{m}$.

resulting in podocyte loss and the development of nephrotic syndrome in podocyte-specific Tsc1-deficient mice at 3 to 4 weeks of age $(19,20)$. In addition, overexpression of pathogenic mutations of laminin $\beta 2$ (Lamb2), $\alpha$ actinin 4 (Actn4), or collagen IV (Col4a3) activates the UPR in podocytes (11, 21-23). In the case of Lamb2, mutation to arginine (C321R) at cysteine residue 321 causes podocyte cell injury and/or nephrotic syndrome in transgenic mouse models $(11,21)$. However, deletion of the IRE1 $\alpha /$ XBP1 pathway (either Ire1a or Xbp1) in the UPR or of Atg5 in autophagy in podocytes has little to no impact on FP or renal function during the first year of life in mice and only caused mild albuminuria after 1 year of age (24-26). These data suggest that IRE1 $\alpha$ of the UPR and autophagy are largely dispensable for podocyte development and function in adult mice under basal conditions.

These findings beg the important question of how ER homeostasis is maintained in podocytes, if the IRE1 $\alpha / \mathrm{XBP} 1$ pathway or autophagy is dispensable. ERAD is the principal quality control mechanism responsible for the recruitment and retrotranslocation of (misfolded) ER-resident proteins for proteasomal degradation (27-29). The SEL1L-HRD1 complex represents the most conserved ERAD branch, with SEL1L being an obligatory cofactor for the E3 ligase HRD1 (30-33). Recent studies have provided compelling evidence for the pathophysiological significance of SEL1LHRD1 ERAD in many cell types including hepatocytes, neuroendocrine cells, adipocytes, immune cells, intestinal epithelial cells, and pancreatic acinar and $\beta$ cells $(27,34-49)$. Indeed, SEL1LHRD1 ERAD plays a key role in normal physiology such as food intake regulation, water homeostasis, and energy metabolism in a substrate-specific manner $(27,28,34)$. In many of these cellular systems, cells seem capable of adapting to SEL1L-HRD1 ERAD deficiency, and the effect of SEL1L-HRD1 ERAD deficiency on cellular function may be uncoupled from ER stress or cell death, presumably (in part) because of the adaptive upregulation of ER chaperones, compensatory activation of other ERAD machineries, and/or protein aggregation that attenuates proteotoxicity of misfolded proteins $(27,28)$.

Here, we report a key role for SEL1L-HRD1 ERAD in podocytes in the pathogenesis of podocytopathy and congenital nephrotic syndrome. Indeed, unlike Ire1a-deficient mice, mice with podocyte-specific Sel1L deficiency develop podocytopathy and severe congenital nephrotic syndrome shortly after weaning. SEL1L-
HRD1 ERAD causes this pathogenesis, at least in part, by degrading misfolded nascent nephrin protein in the ER, which represents a key regulatory step in the formation of the slit diaphragm.

\section{Results}

SEL1L-HRD1 is expressed in human podocytes. We first determined the gene expression pattern of SEL1L-HRD1 ERAD in kidneys using single-cell RNA sequencing (scRNA-Seq) analysis. A total of 2545 human cells collected from a normal kidney sample $(50,51)$ were analyzed and led to the identification of 16 cell populations, of which only approximately $1 \%-2 \%$ of total cells were podocytes (Supplemental Figure 1A; supplemental material available online with this article; https://doi.org/10.1172/JCI143988DS1). Both SEL1L and HRD1 mRNA were ubiquitously detected in many cell types including podocytes and tubular cells, as were the UPR sensor IRE1 (encoded by ERN1) and the ER chaperone BiP (encoded by HSPA5) (Supplemental Figure 1, B-E). We made similar observations upon the analysis of previously published scRNA-Seq data on 24 human kidneys (ref. 52 and data not shown).

To detect SEL1L protein level in kidneys, we generated a SEL1L antibody using a recombinant human SEL1L fragment (amino acids 23-194; ref. 43). In glomeruli of healthy kidneys, we detected SEL1L protein in podocytes that we identified using the podocyte marker nuclear Wilms' tumor 1 (WT1) proteins (asterisks, Figure 1A). In addition, we also detected SEL1L in distal tubular cells (arrowheads, Figure 1A), although this still needed to be confirmed using celltype-specific markers. We observed a similar expression pattern for HRD1 in the kidneys (Figure 1B). As HRD1 stability and function depend on SEL1L $(35,53,54)$, we concluded that the SEL1L-HRD1 ERAD complex is expressed in podocytes of human kidneys.

SEL1L-HRD1 expression in podocytes of patients with nephrotic syndrome. Focal segmental glomerulosclerosis (FSGS) represents a common form of nephrotic syndrome, with initial podocyte injury followed by FP effacement, and can be caused by a variety conditions (55). We next tested whether SEL1L-HRD1 expression was altered in podocytes of 3 patients with FSGS, with 2 "healthy" donor samples as controls. It is important to note that demographic information (e.g., age, race, sex, etc.), the cause and severity of the disease, and medications were unknown to the researchers. We randomly assigned the individuals to 2 experimental groups: healthy1-FSGS1 and healthy2-FSGS2- 
A

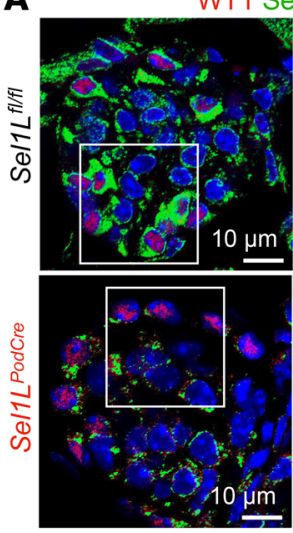

D

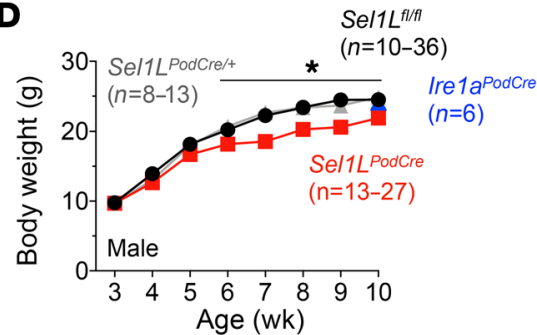

E

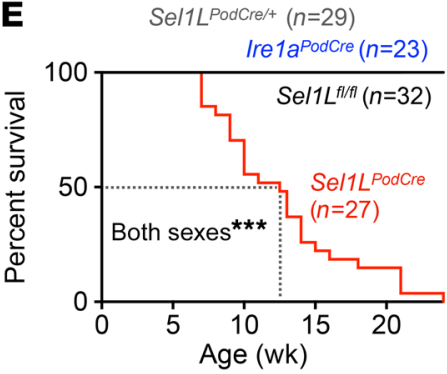

B
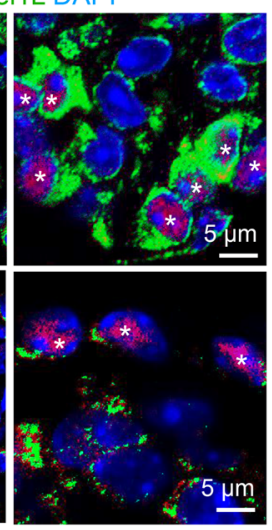

Sel1L fl/fl Ire1a PodCre $(n=6)$

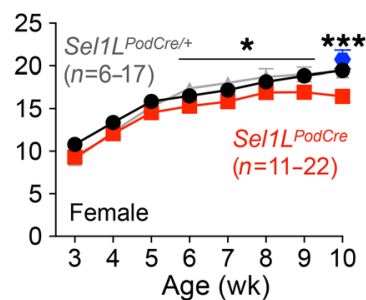

Ire1aPodcre

$(n=6)$

$\mathrm{Se} / 1 L^{\mathrm{fl} / f l}$

$(n=12-20)$

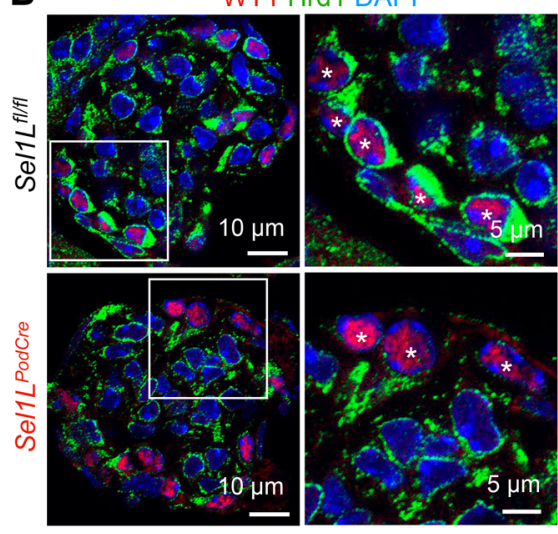

Age (wk)

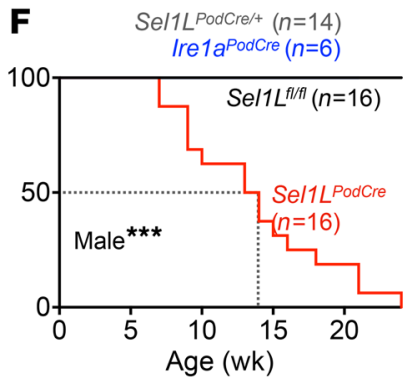

C

O Sel1L filf

$\square$ Sel1LPodCre

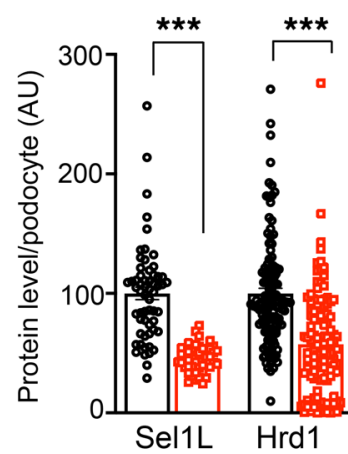

Sel1L Hrd1

Figure 2. Sel1L deficiency in podocytes leads to premature lethality. (A-C) Representative confocal images of SEL1L (A) and HRD1 (B) costaining with WT1 in kidney tissues from 3-week-old Sel1 $L^{f l f l}$ and Sel1L Podcre mice $(n=3$ mice each), with quantitation shown in C $(n=59,45,130$, and 119 podocytes from left to right). Asterisks in the images indicate WT1+ podocytes. Scale bars: $10 \mu \mathrm{m}$ and $5 \mu \mathrm{m}$ (enlarged insets). ${ }^{* * *} P<0.001$, by 2-tailed Student's $t$ test. (D) Growth curves of male and female WT Sel1 $\mathrm{L}^{\mathrm{fl} / \mathrm{fl}}$, heterozygous Sel1 $\mathrm{L}^{\text {Podcrel+}}$, and knockout Sel1 $\mathrm{L}^{\text {Podcre }}$ mice. Ten-week-old $/$ re $1 \alpha^{\text {Podcre }}$ mice were included as a control. ${ }^{*} P<0.05$ and ${ }^{* *} P<0.001$, by 1 -way ANOVA for each age. (E-C) Kaplan-Meier survival analysis for combined (E), male (F), and female (C) sexes. ${ }^{* * *} P<0.0001$, by log-rank test comparing Sel1L ${ }^{\text {Podcre }}$ mice with other cohorts. Values represent the mean \pm SEM.

FSGS3. Participants in both groups were prepared at the same time and imaged under the same settings. In 1 patient (FSGS1), SEL1L and HRD1 protein levels in podocytes were significantly decreased when compared with levels in a healthy individual (Healthy1, Supplemental Figure 2, A and B, and quantitated in Supplemental Figure 2, C and D). By contrast, in 2 other patients (FSGS2 and FSGS3), SEL1L protein levels seemed to be elevated compared with levels in another healthy individual (healthy2), whereas HRD1 protein levels were unchanged compared with those in another healthy individual (Healthy2; Supplemental Figure 2, A and B and quantitated in Supplemental Figure 2, C and D). Although a much larger sample size would be needed to conclude how the expression of ERAD in podocytes changes with disease initiation and progression, these observations suggested a possible role of SEL1L-HRD1 ERAD in podocytes.

Premature lethality of Sel1 $L^{\text {PodCre }}$ mice at approximately 3 months of age. In mouse glomeruli, SEL1L and HRD1 proteins were also expressed in $\mathrm{WT1}^{+}$podocytes (asterisks, Figure 2, A and B). To explore the importance of SEL1L-HRD1 ERAD in podocytes, we generated podocyte-specific SEL1L-deficient mice (Sel1L ${ }^{\text {Podcre }}$ ) by crossing Sel1L-floxed (Sel1L $L^{f / f l}$ ) mice (35) with Cre recombinasetransgenic mice under the control of the highly podocyte-specific promoter podocin, which becomes active during nephrogenesis in newborn kidneys (56). WT Sel1L ${ }^{f l / f l}$ and heterozygous Sel1L ${ }^{\text {PodCre/+ }}$ littermates were included as controls. To assess the relative importance of ERAD versus IRE1 $\alpha$ in podocytes, we generated podocyte-specific Ire1a-deficient mice (Ire $1 a^{\text {PodCre }}$ ) and performed a sideby-side comparison with age- and sex-matched Sel1L ${ }^{\text {PodCre }}$ mice.

We found that SEL1L was specifically deleted in $\mathrm{WT} 1^{+}$podocytes, as demonstrated using immunofluorescent colabeling of SEL1L and WT1 (asterisks, Figure 2A). In keeping with the notion that SEL1L is required for HRD1 protein stability (35), SEL1L deletion also led to reduced HRD1 protein levels in podocytes (asterisks, Figure 2B). Quantitation of SEL1L and HRD1 protein levels in podocytes is shown in Figure 2C. For the first several weeks after weaning, Sel1L $L^{\text {PodCre }}$ mice of both sexes appeared largely normal in size when compared with their 

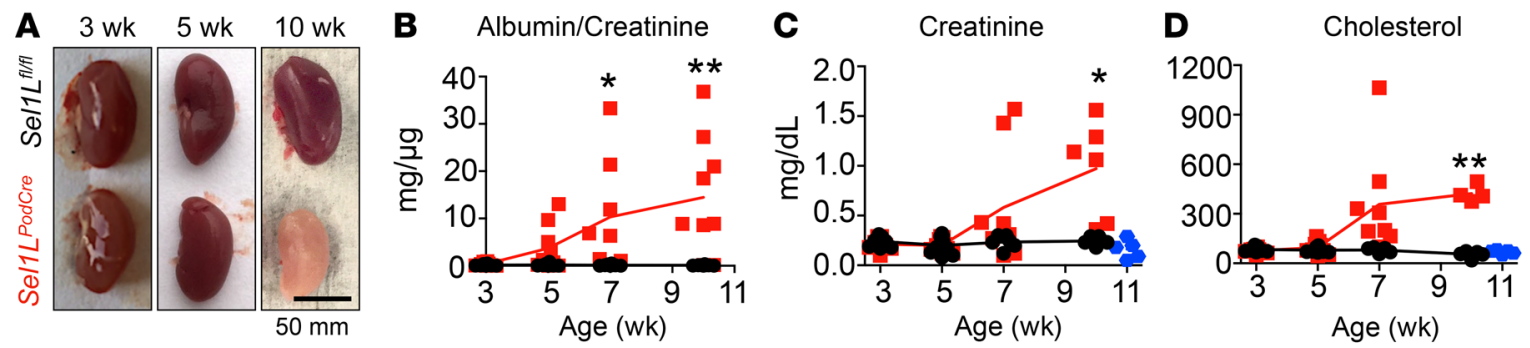

$\mathrm{Sel} / L^{\mathrm{fl} / \mathrm{fl}}$

Sel1LPodCre Ire1aPodCre
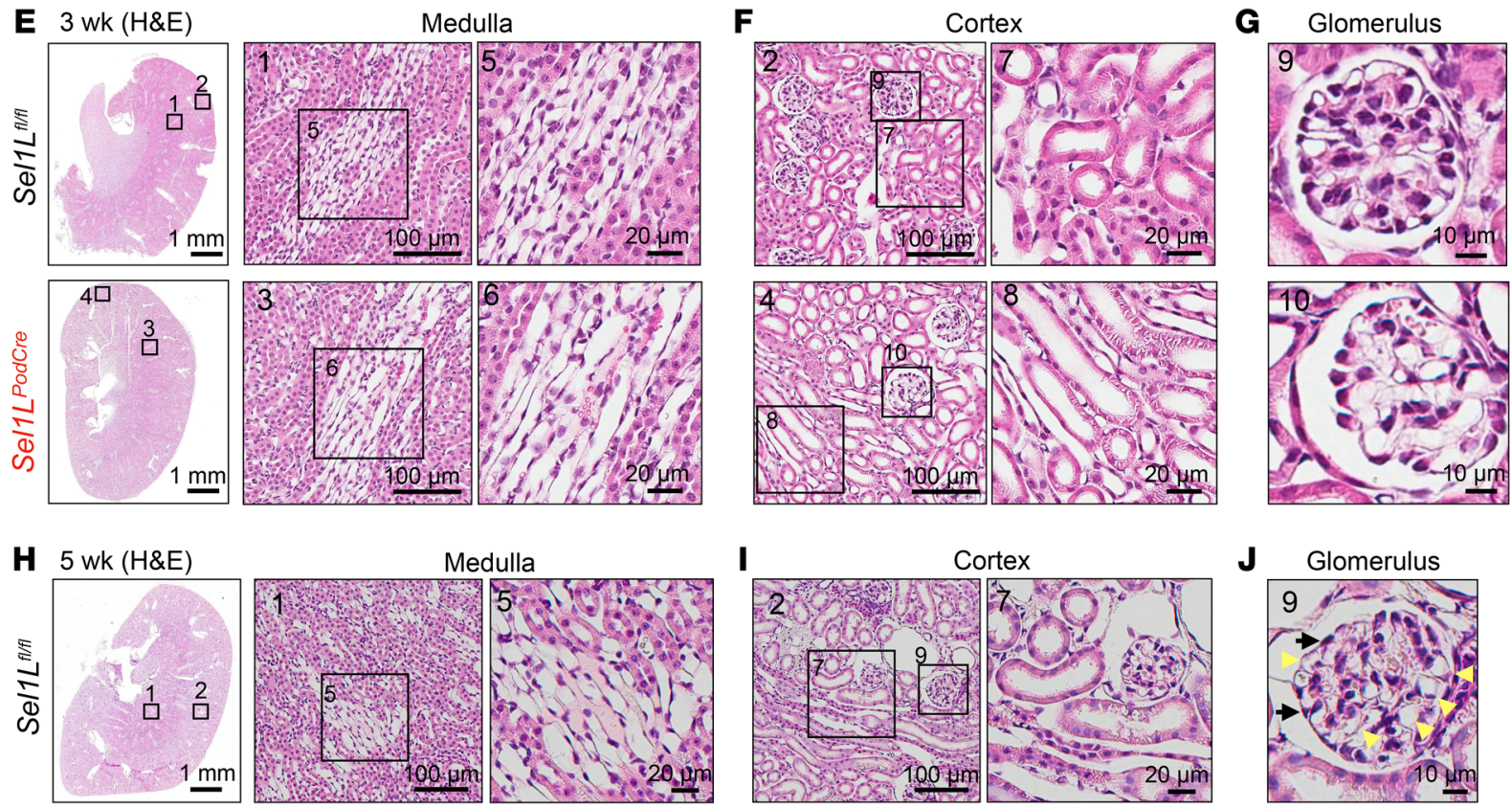

Cortex
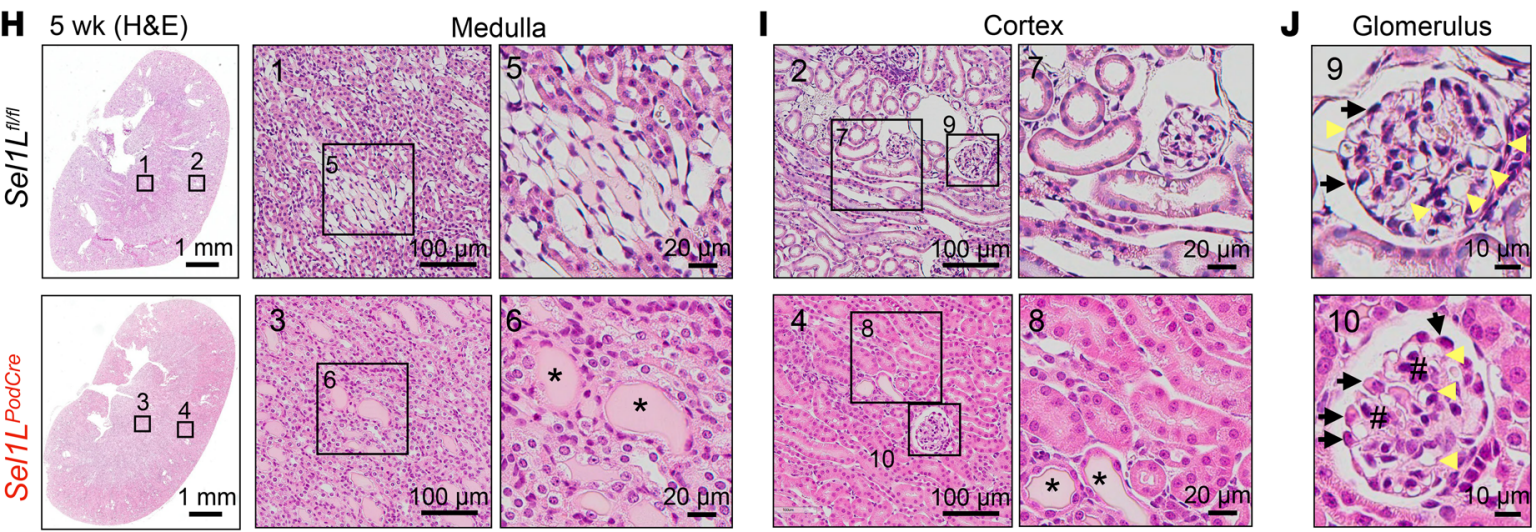

Figure 3. Sel1 ${ }^{\text {Podcre }}$ mice exhibit early-onset renal failure starting at $\mathbf{5}$ weeks of age. (A) Representative images of kidney tissue from 3-, 5-, and 10-weekold Sel1L fl/fl and Sel1L ${ }^{\text {Podcre }}$ mice ( $n=6$ each). (B-D) Ratio of albumin/creatinine in the urine (B) $(n=8$ each at 3 weeks; $n=8-9$ each at 5 weeks; $n=8$ each at 7

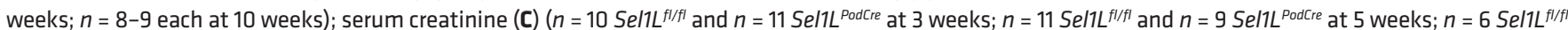

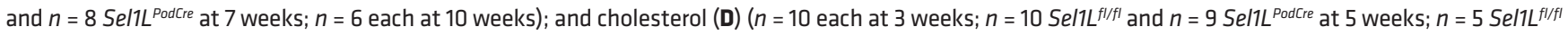
and $n=8 \mathrm{Sel} / 1 L^{\text {PodCre }}$ at 7 weeks; and $n=5 \mathrm{Sel} / \mathrm{L}^{\mathrm{fl} / \mathrm{fl}}$ and $n=4 \mathrm{Sel} / 1 \mathrm{~L}^{\text {Podcre }}$ at 10 weeks). Ten-week-old Ire1a ${ }^{\text {Podcre }}$ mice were included as a control for $\mathbf{C}$ and $\mathbf{D}$ ( $n=5$ and $n=6$ Ire1 $a^{\text {Podcre }}$ mice for $\mathbf{C}$ and $\mathbf{D}$, respectively). Values represent the mean \pm SEM. ${ }^{*} P<0.05$ and ${ }^{* *} P<0.01$; a 2-tailed Student's $t$ test and 1 -way ANOVA were used for data for 3-7 weeks and 10 weeks, respectively. (E-J) Representative H\&E-stained images of kidney sections from 3-week-old (E-C) and 5-week-old $(\mathbf{H}-\mathbf{J})$ mice $(n=3)$. Asterisks indicate protein casts, pound signs indicate mesangial cell hyperplasia, black arrows indicate podocytes, and yellow arrowheads indicate the capillary lumen. Scale bars: $50 \mathrm{~mm}(\mathbf{A}) ; 1 \mathrm{~mm}, 100 \mu \mathrm{m}$, and $20 \mu \mathrm{m}(\mathbf{E}, \mathbf{F}, \mathbf{H}$, and I); and $10 \mu \mathrm{m}$ (G and J).

WT Sel1L $L^{f / f l}$ littermates (Figure 2D). However, starting from 6 weeks of age, Sel1L ${ }^{\text {PodCre }}$ mice began to lose body weight as they became sick (Figure 2D). Sel1L ${ }^{\text {PodCre }}$ mice had a median lifespan of approximately 13 weeks, with a 14-week and 10-week lifespan for males and females, respectively (Figure 2, E-G). By contrast, Ire1a ${ }^{\text {PodCre }}$ mice appeared normal in terms of growth and lifespan compared with WT littermates within the first 6 months of life (blue, Figure 2, D-G), suggesting that IRE1 $\alpha$ of the UPR is dispensable for podocyte function during this time period. For clarity, only the 10-week time point is shown for Ire1 $a^{\text {Podcre }}$ mice in Figure 2D. Moreover, heterozygous Sel1L $L^{\text {PodCre/+ }}$ littermates appeared normal, suggesting that in podocytes, 1 copy of SEL1L is suf- ficient for ERAD function (gray, Figure 2, D-G). Hence, we concluded that podocyte-specific SEL1L-deficient mice die prematurely, with a median lifespan of approximately 13 weeks.

Congenital nephrotic syndrome and renal failure of Sel1L $L^{\text {Podcre }}$ mice. We next addressed how podocyte-specific deletion of SEL1L leads to early lethality in mice. At 3 to 5 weeks of age, Sel1L $L^{\text {PodCre }}$ kidneys appeared normal in color but became pale at 10 weeks of age (Figure 3A). Starting at 5 weeks of age, Sel1L $L^{\text {PodCre }}$ mice exhibited proteinuria, as demonstrated by the presence of albumin in the urine (Supplemental Figure 3A and quantitated in Supplemental Figure 3B; see complete unedited blots in the 
A
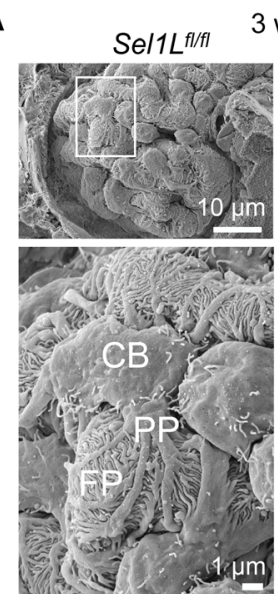

D

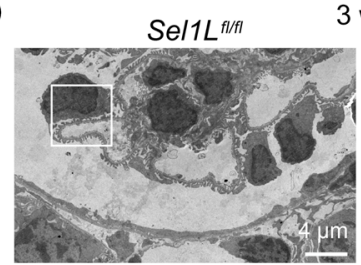

3 wk
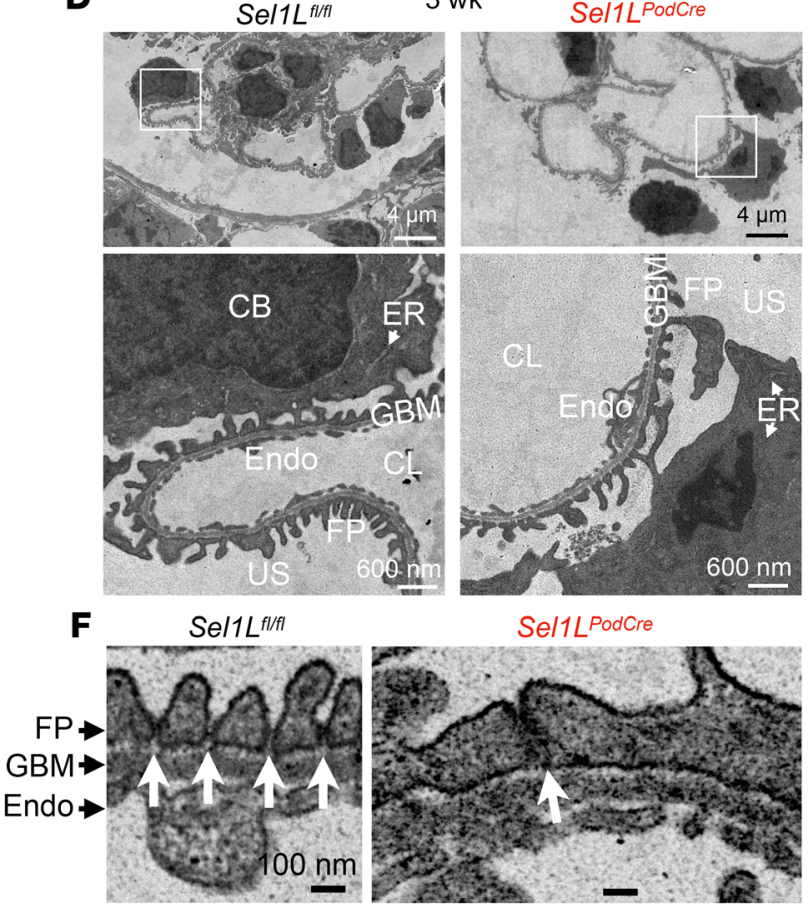

G

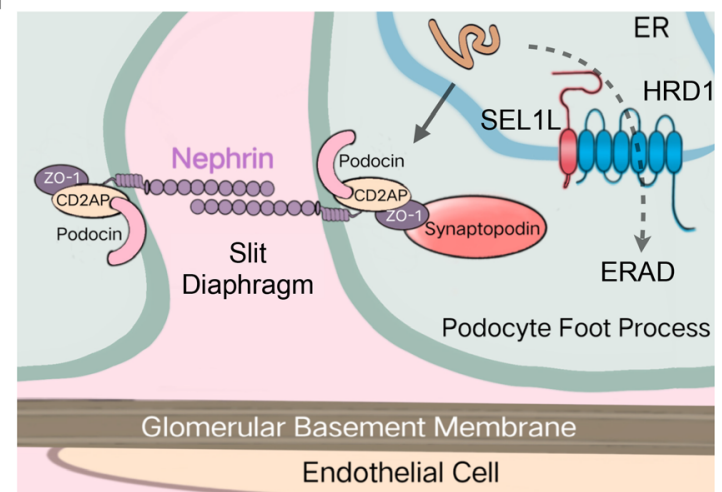

B

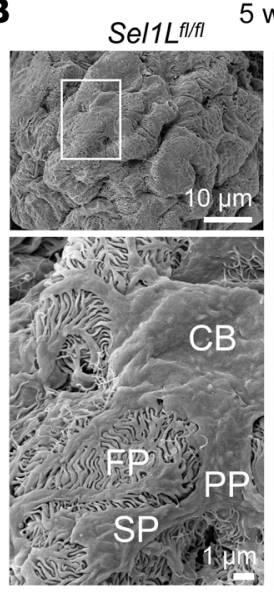

E

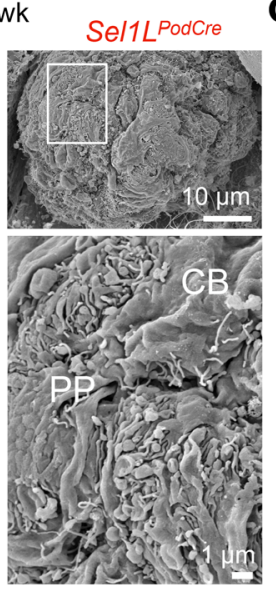

C $\operatorname{Sel} 1 L^{f / \mid f l} 10 \mathrm{wk}$ Sel1LPodCre
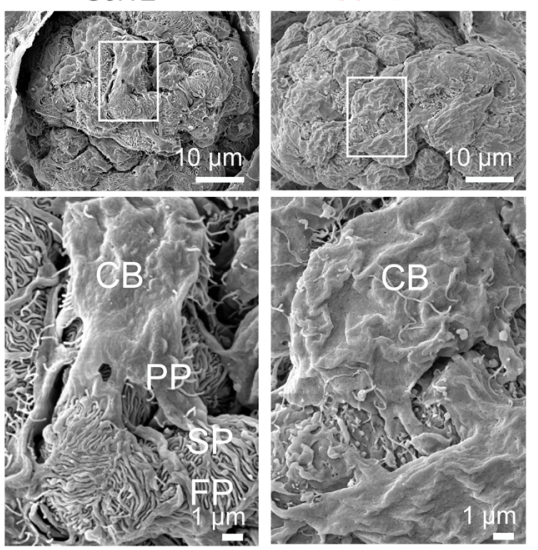

5 wk
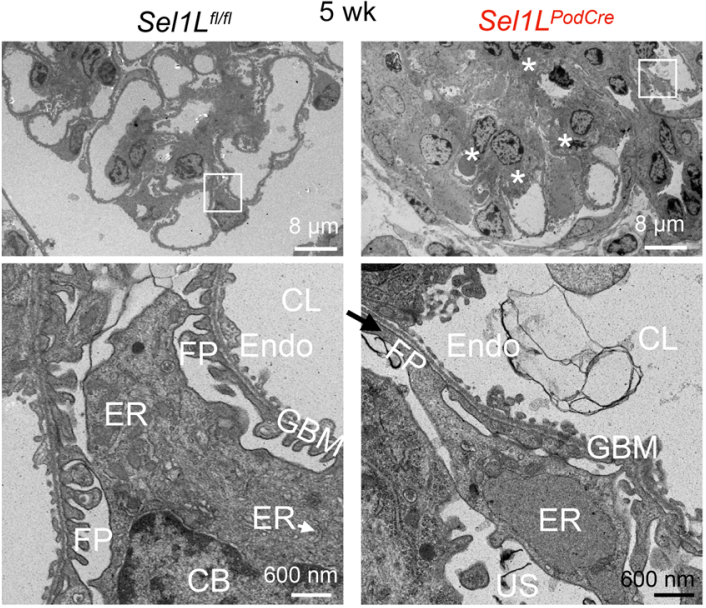

H

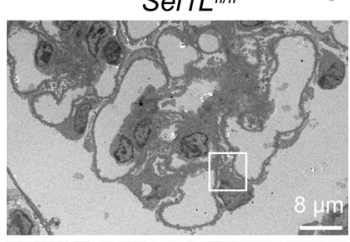

Sel1LPodCre
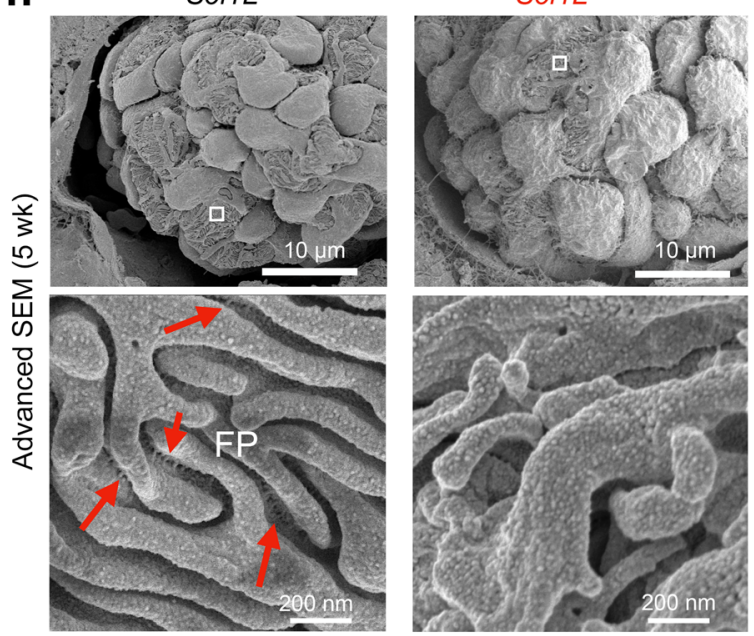

Figure 4. SEL1L is required for the formation of the slit diaphragm. (A-C) Representative SEM images of glomeruli from 3-week-old mice (A) ( $n=9$ Sel1L $L^{f / f l}$ and

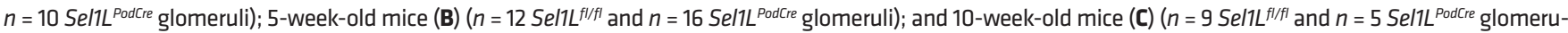
li). $n=2$ mice/genotype. Scale bars: $10 \mu \mathrm{m}$ and $1 \mu \mathrm{m}$ (enlarged insets). (D and E) Representative TEM images of glomeruli from 3-week-old mice (D) ( $n=3$ glomeruli each) and 5-week-old mice (E) ( $n=6$ glomeruli each). $n=2$ mice/genotype. Asterisks indicate mesangial cell hyperplasia; arrows indicate FP fusion. Scale bars: $4 \mu \mathrm{m}(\mathbf{D}), 8 \mu \mathrm{m}(\mathbf{E})$, and $600 \mathrm{~nm}$ (enlarged insets in $\mathbf{D}$ and $\mathbf{E})$. (F) Representative TEM images of slit diaphragms (white arrows). Scale bar: $100 \mathrm{~nm}$. (C) Diagram illustrating the key proteins involved in the slit diaphragm and ERAD. (H) Representative images of advanced SEM images showing slit diaphragms (red arrows) in 5-week-old mice ( $n=7$ glomeruli each). $n=2$ mice/genotype. Scale bars: $10 \mu \mathrm{m}$ and $200 \mathrm{~nm}$ (enlarged insets). CB, cell body of podocytes; CL, capillary lumen; US, urinary space; Endo, endothelial cells. 
A Nephrin KDEL DAPI
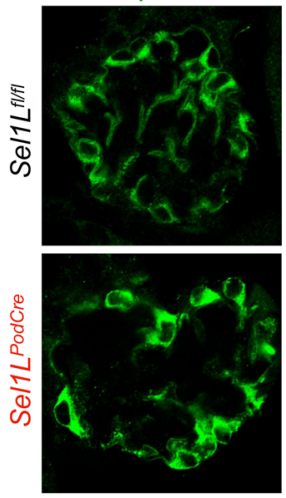

C
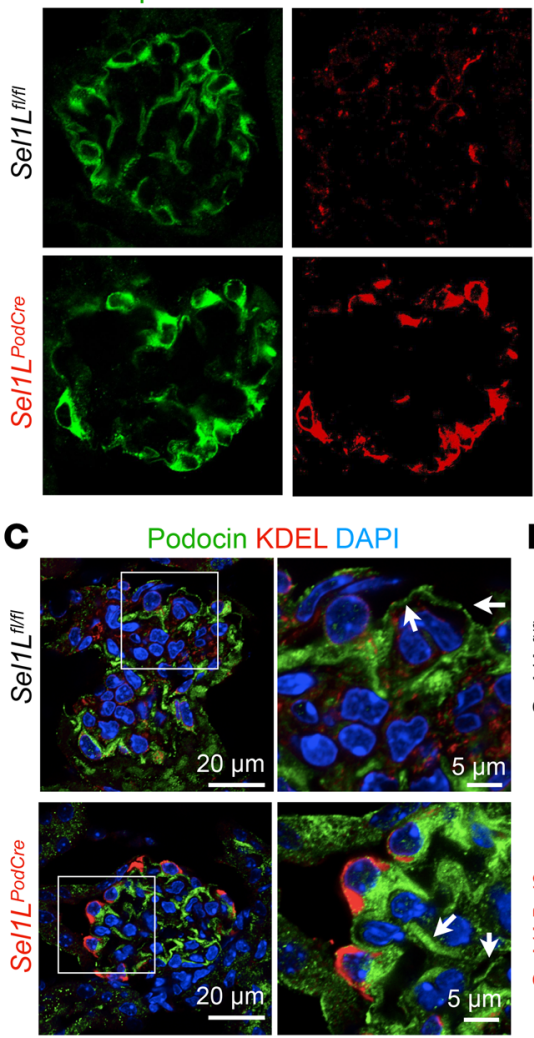

F
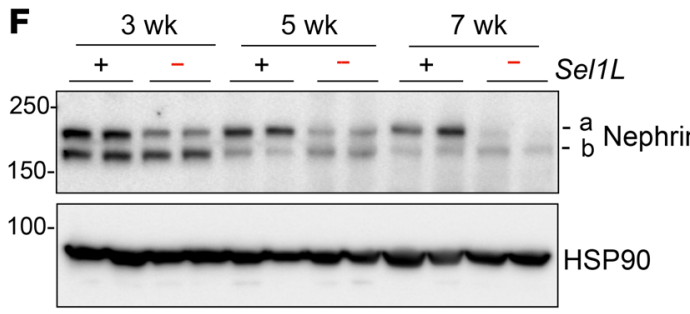
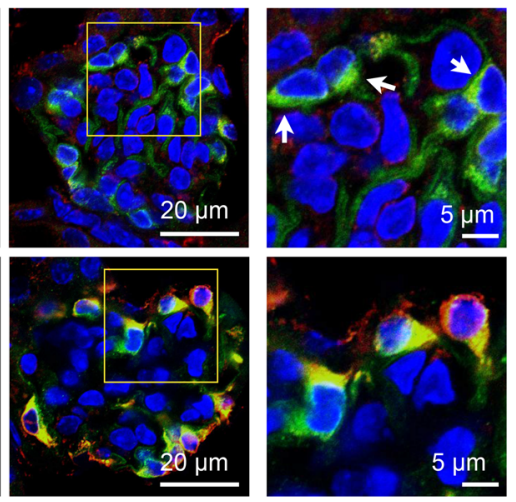

D
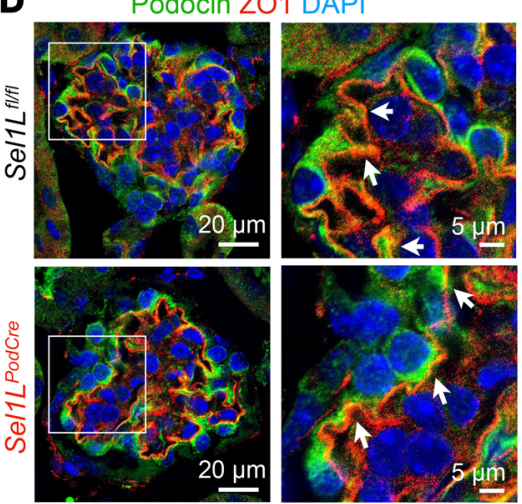

G

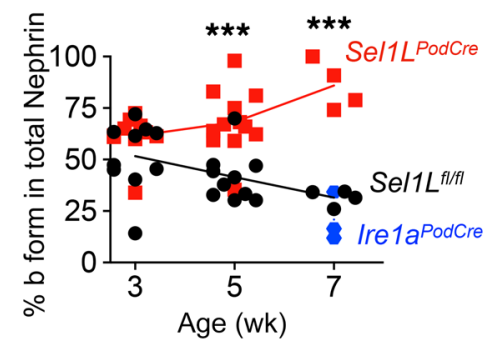

B Nephrin ZO1 DAPI
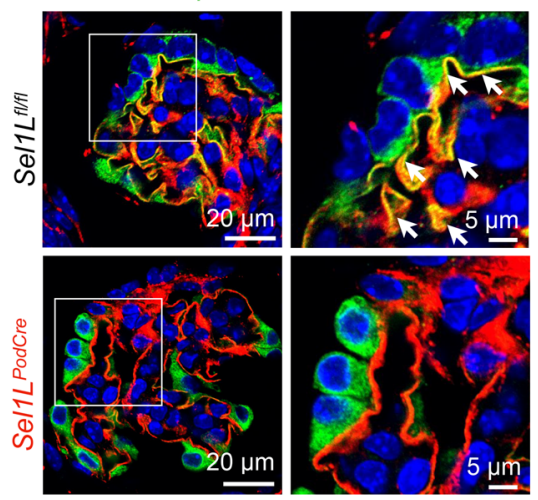

E
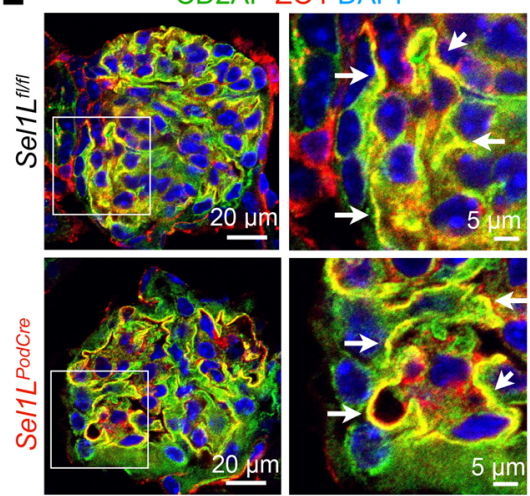

H $5 w k$
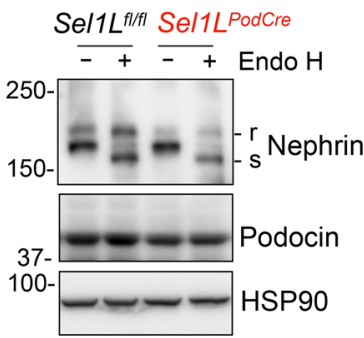

Figure 5. SEL1L deficiency affects the maturation of nascent nephrin in the ER. (A-E) Representative confocal images of nephrin-KDEL (A), nephrin-ZO1 (B), podocin-KDEL (C), podocin-ZO1 (D), and CD2AP-Z01 (E) in kidney sections from 3-week-old mice ( $n=3$ mice each). Arrows mark the perinuclear localization of nephrin (A), colocalization of nephrin with ZO1 (B), localization of podocin at the slit diaphragm (C), and colocalization of podocin and Z01 (D) and CD2AP and ZO1 (E). Images with artificially enhanced KDEL signal are shown in Supplemental Figure 6A. (F) Western blot analysis of nephrin in kidney lysates from 3-, 5-, and 7-week-old mice and (G) quantitation of the percentage of $b$ form nephrin in total nephrin. $n=10$ mice each at 3 weeks; $n=10 \mathrm{Sel} / \mathrm{L}^{\mathrm{fl} / \mathrm{fl}}$ and $n=12 \mathrm{Sel} / 1 \mathrm{~L}^{\text {PodCre }}$ at 5 weeks; and $n=4$ each at 7 weeks. Seven-week-old Ire1a ${ }^{\text {Podcre }}$ mice were included as a control ( $\left.n=3\right)$, and the original data are shown in Supplemental Figure 7A. Values represent the mean \pm SEM. ${ }^{* *} P<0.001$, by 2-tailed Student's $t$ test (3-and 5-week-old mice) and 1-way ANOVA (7-week-old mice). (H) Western blot analysis of nephrin in EndoH-treated kidney lysates from 5-week-old mice, with quantitation shown in Supplemental Figure 6D ( $n=5$ mice/group). r, EndoH-resistant form; s, EndoH-sensitive form.

supplemental material). The ratio of albumin to creatinine in the urine of Sel1L $L^{\text {Podcre }}$ mice was elevated starting at 5 weeks of age when compared with ratios in WT littermates (Figure 3B). Similarly, blood concentrations of creatinine, cholesterol, and blood urea nitrogen (BUN), indicators of kidney function, were elevated starting at 7 weeks of age in Sel1L $L^{\text {PodCre }}$ mice (Figure 3, C and D, and Supplemental Figure 3C). However, the level of serum alanine aminotransferase (ALT), an indicator of liver function, was unchanged with age, even at 10 weeks, when many Sel1L $L^{\text {PodCre }}$ mice were moribund (Supplemental Figure 3D), thus excluding the possible contribution from secondary liver damage in the disease pathogenesis. By contrast, 10-week-old Ire $1 a^{\text {PodCre }}$ mice appeared normal in terms of proteinuria, serum levels of creatinine, cholesterol, and BUN (Figure 3, C and D, and Supplemental Figure 3, B, C, and E).

Histochemical examination of the kidneys from 3-week-old cohorts revealed normal morphology of the kidney medulla, cortex, and glomeruli (Figure 3, E-G). However, at 5 weeks of age, we observed that renal tubules in both the cortex and medulla were filled with large protein casts in Sel1L $L^{\text {PodCre }}$ mice (asterisks in highlighted boxes 5 vs. 6 and 7 vs. 8, Figure 3, H and I). Moreover, although the glomeruli sizes were similar between the cohorts, 
A

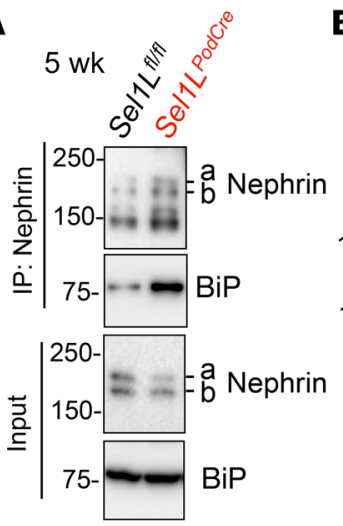

E

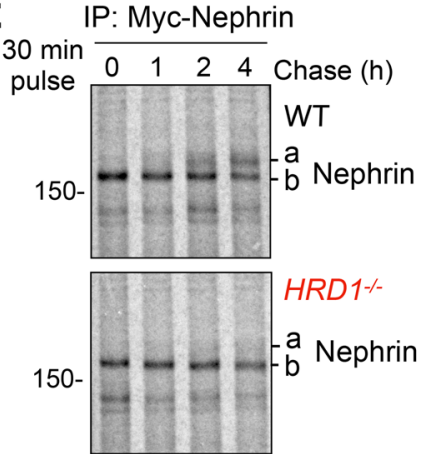

H

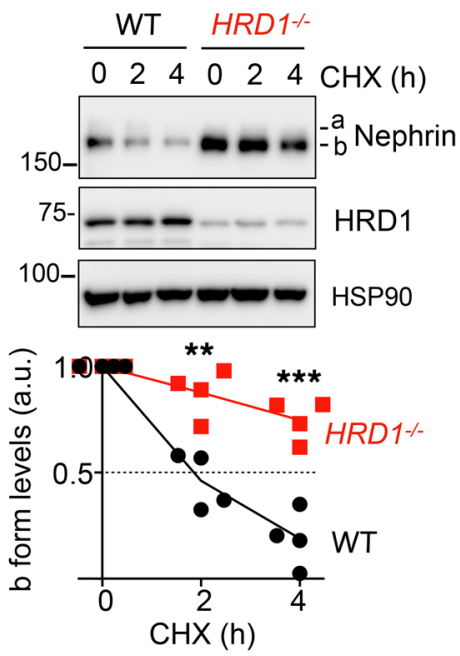

B

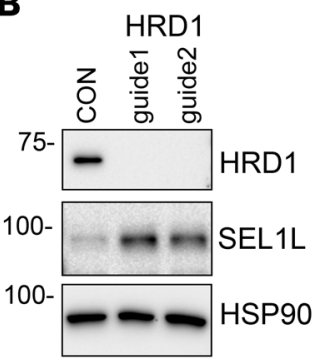

Human podocytes

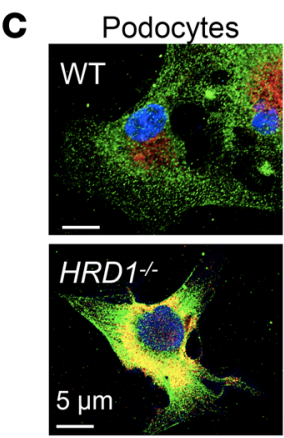

NEPHRIN KDEL DAPI
D

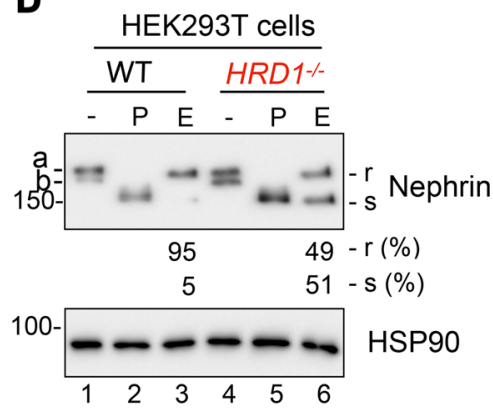

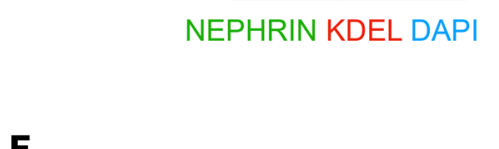
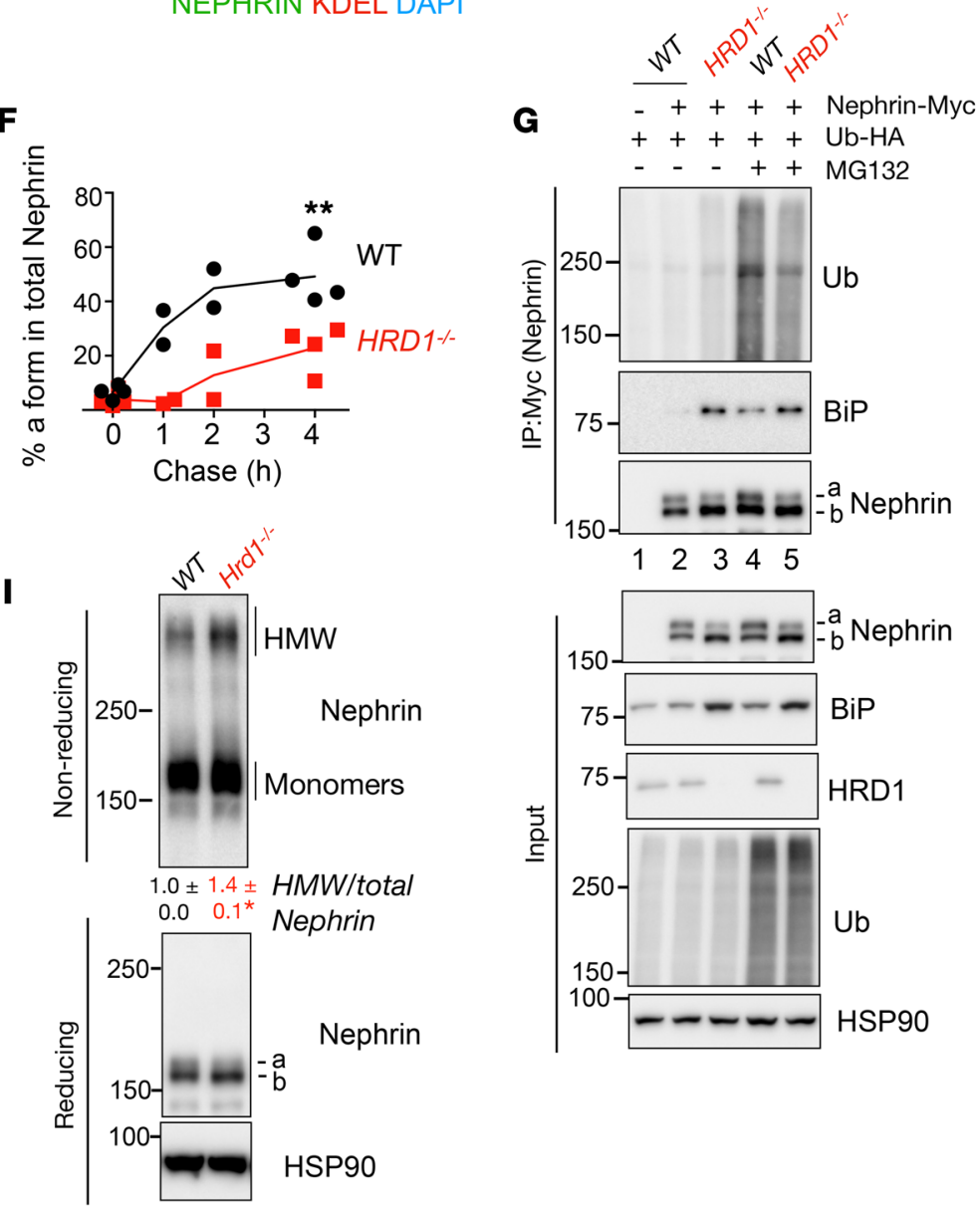

Figure 6. Nephrin is an endogenous substrate of ERAD, and in the absence of ERAD, nephrin is retained in the ER and associated with BiP. (A) Western blot analysis following nephrin immunoprecipitation in kidney tissues from 5-week-old mice, showing the interaction between nephrin and BiP in the absence of ERAD. (B) Western blot analysis following HRD1 deletion in the HRD1-/- human podocyte line. CON, control. (C) Representative confocal images of nephrin and KDEL staining

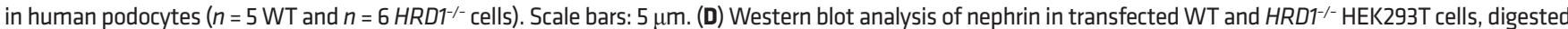
with or without PNGase $F(P)$ or EndoH (E), with quantitation of the percentage of EndoH-resistant and EndoH-sensitive forms shown below. (E) ${ }^{35} \mathrm{~S}$ pulse (30-min) chase (0, 1, 2, and 4 hours) analysis of nascent nephrin protein in HEK293T cells, and (F) quantitation of the percentage of $a$ form nephrin in total nephrin. (G) Western blot analysis of Myc immunoprecipitates in transfected HEK293T cells, treated or not with $10 \mu \mathrm{M}$ MG132 for 5 hours prior to harvesting, showing ERAD-mediated ubiquitination of nephrin. (H) Western blot analysis of nephrin protein decay in transfected HEK293T cells treated with brefeldin A and/or CHX for the indicated durations, with quantitation from 4 independent experiments shown below. (I) Western blot analysis of nephrin in transfected WT and Hrd1/- N2a cells under nonreducing or reducing conditions, with the level of HMW nephrin normalized to total nephrin from 3 independent experiments shown below the blot. Data are representative of at least 3 independent experiments. Values represent the mean $\pm \mathrm{SEM}$. ${ }^{*} P<0.05$, ${ }^{*} P<0.01$, and ${ }^{* * *} P<0.001$, by 2 -tailed Student's $t$ test.

Sel1L PodCre glomeruli showed mesangial cell hyperplasia (indicated by pound signs), with reduced open capillary loops (yellow arrowheads, highlighted boxes 9 and 10, Figure 3J). Quantitation of glomeruli size and mesangial cell hyperplasia is shown in Supplemental Figure 3, F and G. Unlike flattened podocytes at the periph- ery of glomeruli in WT mice, Sel1L PodCre podocytes were rounded (black arrows, highlighted boxes 9 and 10, Figure 3J). Taken together, we concluded that disease initiation in Sel1L PodCre mice occurred around 3 to 5 weeks of age and that SEL1L deficiency in podocytes led to early-onset renal failure and premature death. 

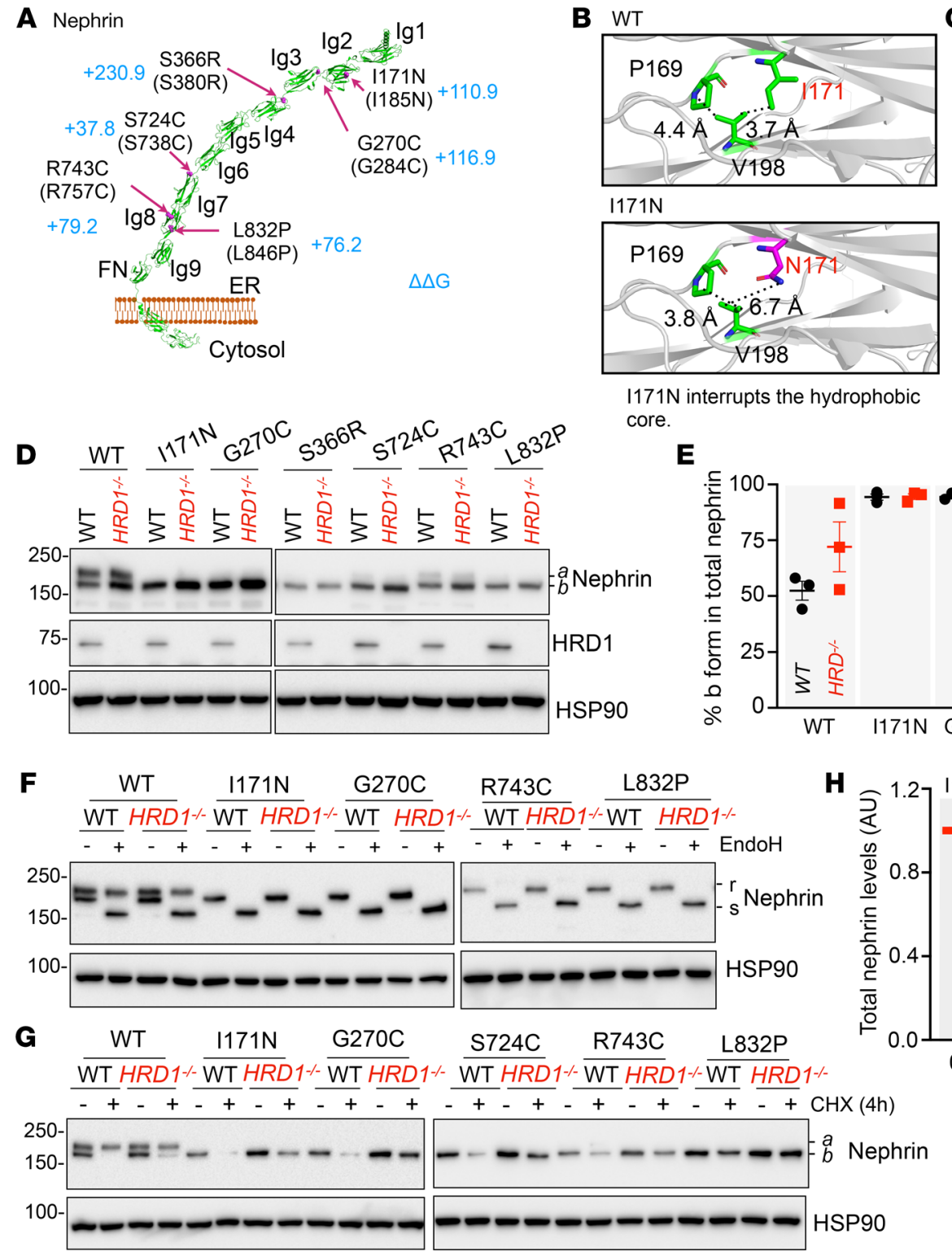

$171 \mathrm{~N}$ interrupts the hydrophobic
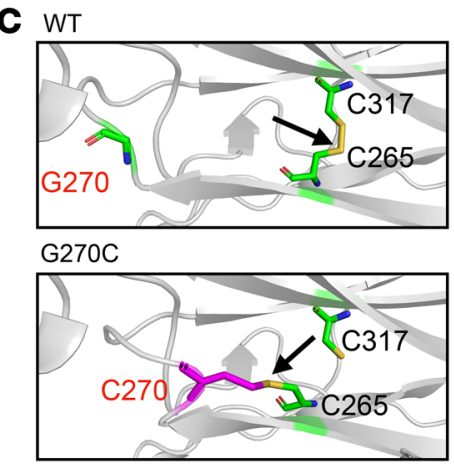

G270C disrupts original disulfide bond between C317 and C265.

I171N G270C S366R S724C R743C L832P

Figure 7. Nephrin disease mutants are unstable, retained in the ER, and targeted for proteasomal degradation by ERAD. (A) Structural modeling of human nephrin showing the domains (Ig and FN) and location of 6 pathogenic mutations. EvoEF2 ( $\Delta \Delta \mathrm{G})$ for each mutant is indicated in blue. (B and C) The predicted local structures of WT, I171N (B), and C270C (C). WT and mutated residues are shown in green and magenta, respectively. Distances between the indicated residues are shown. Arrows indicate disulfide bonds. (D and E) Western blot analysis of WT and mutant nephrin proteins in transfected HEK293T cells, showing nephrin mutants running as the $b$ form on SDS-PAGE. Quantitation is shown in E. (F) Western blot analysis following EndoH digestion in HEK293T cells transfected with WT or mutant nephrin. (G and $\mathbf{H})$ Western blot analysis of WT and mutant nephrin in transfected HEK293T cells treated with $\mathrm{CHX}$ for 4 hours, with quantitation shown in $\mathbf{H}$. Values represent the mean \pm SEM. Data are representative of at least 2 independent experiments. ${ }^{*} P<0.05$, by 2 -tailed Student's $t$ test.

Impaired slit diaphragm in Sel1L ${ }^{\text {PodCre }}$ mice. We next performed scanning electron microscopy (SEM) to visualize glomeruli and podocytes from 3-, 5-, and 10-week-old mice. Images from 2 independent samples are shown for each age in Figure $4, \mathrm{~A}^{-} \mathrm{C}$, and Supplemental Figure 4, A-C. At 3 weeks of age, glomeruli from Sel1 $L^{\text {Podcre }}$ mice appeared generally normal in size, shape, and overall morphology (Figure 4A and Supplemental Figure 4A). Podocytes formed primary and secondary processes (PPs and SPs), as well as interdigitating FPs in Sel1L $L^{\text {PodCre }}$ mice. However, starting at 5 weeks of age, Sel1L $L^{\text {PodCre }}$ mice exhibited broadened
PPs and shortened SPs (Figure 4B and Supplemental Figure 4B). Moreover, interdigitating FPs between podocytes were dramatically disorganized and misdirected, similar to those in nephrin-deficient mice $(8,57,58)$. The defects in FPs became more severe with age in Sel1L $L^{\text {PodCre }}$ mice: by 10 weeks of age, some glomeruli were severely damaged and showed FP effacement (Figure 4C and Supplemental Figure 4C).

We next performed transmission electron microscopy (TEM) to visualize ultrastructural changes of podocytes and the filtration unit at different ages. In healthy glomeruli, 3 layers, the inner- 

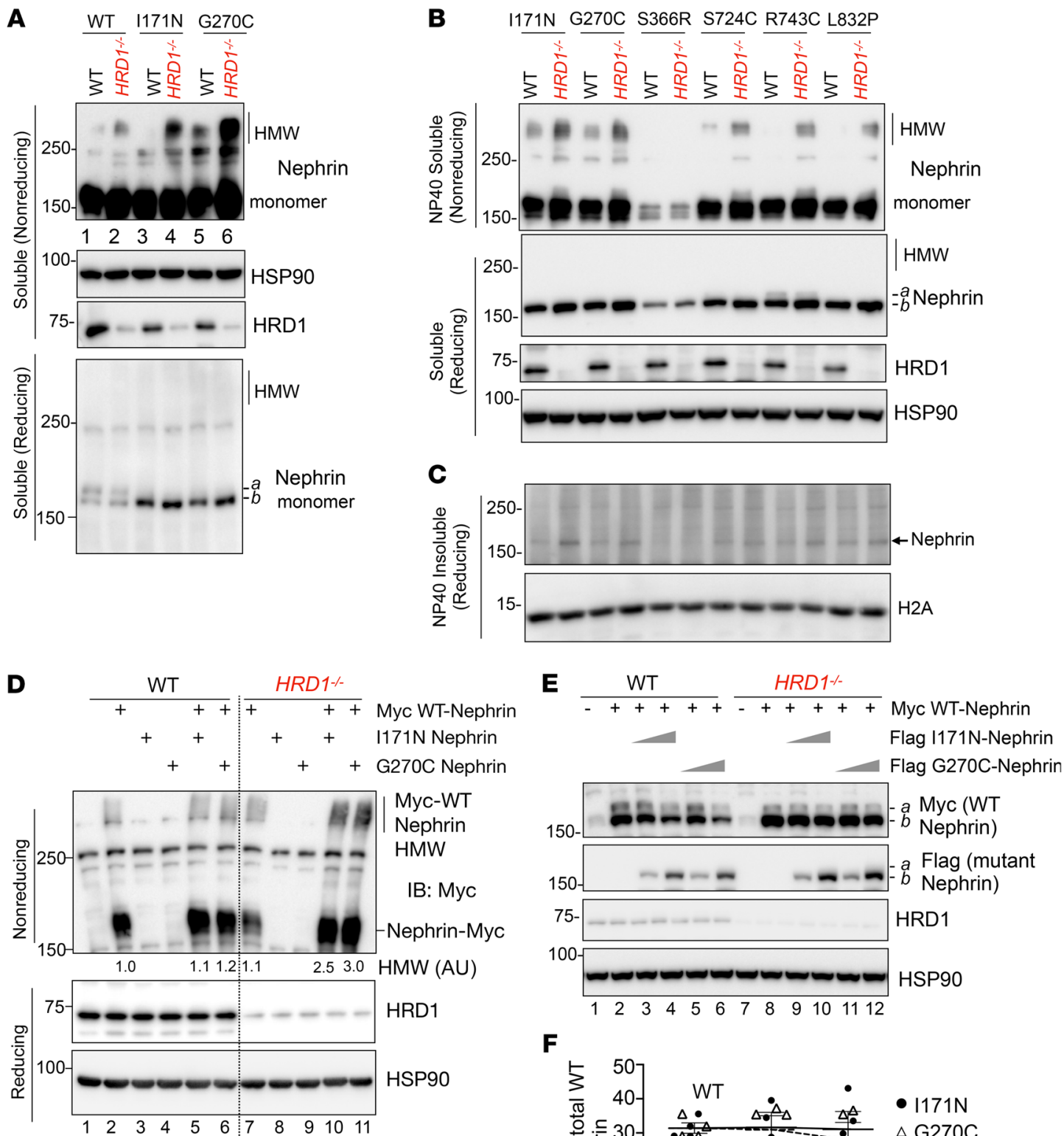

$\mathbf{F}$

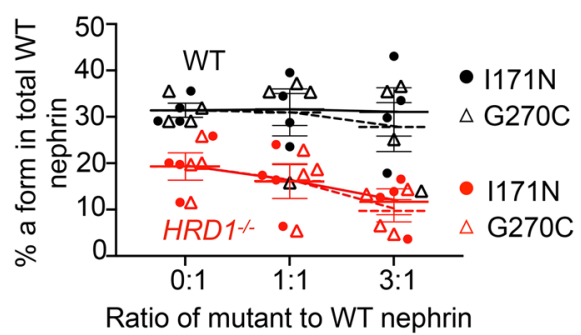

Figure 8. ERAD of disease mutants attenuates their pathogenicity toward the WT allele. (A) Western blot analysis of WT and mutant HMW and monomeric nephrin in transfected WT and HRD1 ${ }^{-1-}$ HEK293T cells under nonreducing and reducing conditions. (B and C) Western blot analysis of NP40-soluble (B) and NP40-insoluble fractions (C) in transfected WT and HRD1-/- HEK293T cells, showing increased formation of HMW and insoluble nephrin aggregates in HRD1-/- HEK293T cells for both WT and mutant nephrin. (D) Western blot analysis of nephrin HMW aggregation in HEK293T cells transfected with different combinations of Myc-WT nephrin and nephrin mutants under nonreducing conditions, with the level of HMW nephrin from 1 representative experiment shown below the blot. (E and F) Western blot analysis of Myc-WT and Flag-mutant nephrin in HEK293T cells transfected with different combinations of Myc-WT nephrin and Flag-tagged mutant nephrin at a 1:1 or 1:3 ratio. Quantitation of the percentage of $a$ form WT nephrin in total WT nephrin is shown in F, indicating a decrease in the percentage of $a$ form WT nephrin in HRD1 $1^{-1-}$ HEK293T cells (upon cotransfection of an increased amount of mutant nephrin) when compared with that in WT HEK293T cells. Values represent the mean \pm SEM. Data are representative of at least 2 independent experiments.

most endothelium, the glomerular basement membrane (GBM), and the podocytes with interdigitating FPs form the glomerular filtration barrier of the kidneys (1). At 3 weeks of age, 3 layers of the filtration unit appeared largely normal (Figure 4D and Supplemental Figure 4D). However, at 5 weeks, Sel1L PodCre $^{\text {mice exhibited }}$ mesangial cell hyperplasia (indicated by asterisks) and fusion of FPs (black arrows, Figure 4E and Supplemental Figure 4E).

Interdigitating FPs are held together by an extracellular structure known as the slit diaphragm (arrows, Figure 4F and diagram shown in Figure $4 \mathrm{G}$ and refs. 2, 3). Quantitation of the length of 
slit diaphragm revealed that the length was approximately $40 \mathrm{~nm}$ in 5-week-old WT mice (Supplemental Figure 4F), consistent with previous reports $(57,59-61)$. On the other hand, Sel1L ${ }^{\text {PodCre }}$ podocytes had very few slit diaphragms, and the junctions between the remaining adjacent FPs were very narrow (arrow, Figure 4F). This was further confirmed using ultra-high-resolution advanced SEM analyses, in comparison with regular SEM on the same podocytes (Supplemental Figure 5A), in which the slit diaphragm was visible as a bridge-like structure linking adjacent FPs in WT mice, but largely absent in Sel1L ${ }^{\text {PodCre }}$ mice at 5 weeks of age (red arrows, Figure $4 \mathrm{H}$ and Supplemental Figure 5, B and C). Hence, we concluded that SEL1L deficiency in podocytes led to impaired slit diaphragms in mice at 5 weeks of age, which coincided with the development of proteinuria and nephrotic syndrome.

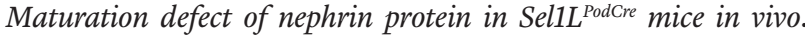
Nephrin, together with other proteins such as the ER protein podocin and the cytosolic proteins CD2AP and synaptopodin, forms the slit diaphragm (Figure 4G). We next explored whether and how SEL1L deficiency in podocytes affects the intracellular localization of these proteins by performing costaining with markers for the ER (KDEL) and the slit diaphragm (ZO1). In WT podocytes, we found that nephrin had a diffused pattern surrounding the nucleus (Figure 5A), with some colocalization with KDEL after artificial saturation of KDEL signal (arrows, Supplemental Figure 6A). In addition, nephrin was distributed basally, adjacent to the GBM along the peripheral capillary loops, as demonstrated by its colocalization with the cell junction protein ZO1 (arrows, Figure 5B). ZO1 continuously distributes along the GBM, as previously shown $(62,63)$. By contrast, we observed that in Sel1L $L^{\text {PodCre }}$ podocytes, the colocalization of nephrin and the ER maker KDEL was significantly increased (Figure 5A), whereas the colocalization of nephrin and ZO1 was markedly decreased (Figure 5B). Of note, KDEL levels were increased in the absence of SEL1L, probably as a result of the cellular adaptive response to ERAD deficiency $(37,38)$.

In direct contrast with nephrin, maturation of another key component of slit diaphragms, podocin (encoded by NPHS2), in the ER appeared to be unaffected in Sel1L $L^{\text {PodCre }}$ podocytes, as they were able to exit the ER (i.e., lack of colocalization with KDEL, Figure $5 \mathrm{C}$ ), with some reaching the GBM along the peripheral capillary loops (i.e., colocalization with ZO1, arrows, Figure 5D). Moreover, distribution of the actin-associated PP markers CD2AP (arrows, Figure 5E) and synaptopodin (arrows, Supplemental Figure 6B) along the GBM was unaffected by Sel1L deficiency.

To further demonstrate biochemically how nephrin maturation is affected by SEL1L-HRD1 ERAD, we next performed various biochemical assays. It is well documented that nephrin proteins appear as 2 bands on SDS-PAGE around $180 \mathrm{kDa}$ - slow-migrating mature $\mathrm{a}$ (nephrin $a$ ) and fast-migrating ER-retained $\mathrm{b}$ forms (nephrin $b$ ) - as a result of differential glycosylation at 10 potential $N$-glycosylation sites $(15,64-66)$. In WT mice, the percentage of nephrin $b$ in total nephrin was approximately $50 \%$ at 3 weeks of age, and decreased with age, reaching $35 \%$ and $25 \%$ at 5 and 7 weeks of age, respectively (Figure $5 \mathrm{~F}$ and quantitated in Figure 5G, uncropped gel available online). By contrast, in Sel1 $L^{\text {PodCre }}$ kidneys, the percentage of nephrin $b$ was mildly elevated, at approximately $60 \%$ at 3 weeks of age, but significantly increased with age, reaching $70 \%$ and $85 \%$ at 5 and 7 weeks of age, respectively (Figure $5 \mathrm{~F}$ and quan- titated in Figure 5G). Of note, at 7 weeks of age, total nephrin protein levels were significantly decreased in Sel1L $L^{\text {PodCre }}$ kidneys (Figure $5 \mathrm{~F}$ ), probably a result of the elevated podocyte loss observed by SEM (Supplemental Figure 4C). Furthermore, in the purified microsomal fractions (containing the ER and other endomembranes) from kidneys of 3-week-old mice, the total amount and percentage of ER-retained $b$ form nephrin was elevated in Sel1L $L^{\text {PodCre }}$ kidneys (Supplemental Figure 6C), providing further support for elevated ER accumulation of nephrin in Sel1L $L^{\text {PodCre }}$ podocytes, as revealed in immunofluorescence staining (Figure 5A).

We next subjected kidney lysates to endoglycosidase $\mathrm{H}$ (EndoH) digestion to distinguish immature high-mannose, EndoH-sensitive forms (i.e., present in the ER) from the EndoH-resistant forms (i.e., mature beyond the ER). The EndoH-sensitive fraction of total nephrin protein was approximately $40 \%$ in WT kidneys versus $60 \%$ in Sel1L ${ }^{\text {PodCre }}$ kidneys at 5 weeks of age (Figure $5 \mathrm{H}$ and quantitated in Supplemental Figure 6D). As controls, the levels of podocin, which has no predicted glycosylation sites, as well as of the cytosolic protein synaptopodin were largely unchanged in Sel1L ${ }^{\text {PodCre }}$ kidneys at different ages (Figure $5 \mathrm{H}$ and Supplemental Figure 6E). Taken together, we concluded that SEL1L deficiency caused defects in the maturation of nephrin in the ER, while having no notable effect on the localization or levels of other major slit diaphragm proteins such as CD2AP, podocin, and synaptopodin.

Normal nephrin maturation in Ire1a-deficient podocytes. We next asked whether nephrin defects are specific to SEL1L deficiency. To this end, we analyzed the status of nephrin protein in Ire1 $a^{\text {PodCre }}$ mice (Supplemental Figure 7). The ratio of $b$ to $a$ forms of nephrin was comparable between Ire1 ${ }^{\text {PodCre }}$ mice and their WT littermates from 3 to 7 weeks of age (Supplemental Figure 7A and quantitated in Figure $5 G$ ). Similarly, podocin and synaptopodin levels were comparable between the cohorts at different ages (Supplemental Figure 7B). Moreover, confocal microscopy revealed that, in Ire $1 a^{\text {PodCre }}$ podocytes, nephrin protein was present around the nucleus and basally adjacent to the GBM along the peripheral capillary loops, similar to what we observed in WT littermate podocytes (arrows, Supplemental Figure 7C). Hence, the nephrin defects in Sel1L $L^{\text {PodCre }}$ mice are uncoupled from IRE1 $\alpha$ of the UPR.

Maturation defect of nascent nephrin protein in ERAD-deficient cells in vitro. To further demonstrate the impact of Sel1L deficiency on nephrin folding, we performed immunoprecipitation of nephrin in 5-week-old kidneys. We observed elevated interaction between nephrin and BiP, a key ER chaperone involved in protein folding and degradation, in Sel1L ${ }^{\text {Podcre }}$ kidneys (Figure 6A). This finding provided a plausible explanation for the delayed maturation and ER accumulation of nephrin in Sel1L-deficient cells and pointed to the effort to (re-)fold nephrin in the absence of ERAD. Hence, we speculated that nephrin may be prone to misfolding and subjected to quality control by SEL1L-HRD1 ERAD.

To further establish the causal relationship between SEL1L deficiency and nephrin maturation, we performed the following experiments in vitro. We first generated HRD1-deficient human podocytes using the CRISPR/Cas9 system (Figure 6B). In line with other cell types $(37,38,43)$, HRD1 deletion in podocytes stabilized SEL1L protein, leading to its accumulation (Figure 6B). In HRD1-deficient human podocytes, nephrin accumulated and was predominantly retained in the ER, similar to Sel1 $L^{\text {PodCre }}$ podocytes (Figure 6C). Next, 
we generated an HRD1-deficient human embryonic kidney $293 \mathrm{~T}$ (HEK293T) cell line, which does not express endogenous nephrin. Similar to endogenous nephrin in kidneys, we found that loss of HRD1 increased the proportion of nephrin $b$ (lane 1 vs. 4 ) as well as the EndoH-sensitive form of nephrin ( $51 \%$ vs. $5 \%$ in WT cells, lane 3 vs. 6, Figure 6D). Overexpression of HRD1 in HRD1-deficient HEK293T cells reversed nephrin maturation defects and increased the percentage of nephrin $a$ (Supplemental Figure 8A). The differential mobility shift in EndoH-treated samples was due to glycosylation, as the EndoH-resistant form was sensitive to PNGase F treatment, which removes almost all $N$-linked oligosaccharides from glycoproteins (lane 2 vs. 5, Figure 6D).

Third, to directly visualize nascent protein maturation in the ER, we performed ${ }^{35} \mathrm{~S}$ metabolic labeling followed by a chase for different time points in Myc-tagged, nephrin-transfected HEK293T cells. Strikingly, nearly $50 \%$ of the nascent nephrin proteins matured into nephrin $a$ within 4 hours in WT cells versus only $20 \%$ in $\mathrm{HRD1}^{-/-}$cells (Figure $6 \mathrm{E}$ and quantitated in Figure 6F). Taken together, these data suggested that SEL1L-HRD1 ERAD is required for the maturation of nascent nephrin protein in the ER.

Nephrin is a bona fide endogenous ERAD substrate. Decreased nephrin maturation and an elevated association with $\mathrm{BiP}$ in ERAD-deficient cells prompted the hypothesis that nephrin is an endogenous ERAD substrate. We first asked whether nephrin is ubiquitinated by SEL1L-HRD1 ERAD. In a gain-of-function assay, we transfected HEK293T cells with nephrin, together with Myc-tagged WT HRD1 or an E3 ligase-dead C2A HRD1 mutant. Indeed, nephrin interacted with HRD1 and was ubiquitinated by HRD1 in an E3 ligase-dependent manner (lane 4 vs. 6, Supplemental Figure 8B). Conversely, in the absence of HRD1, we found that ubiquitination of nephrin was markedly reduced compared with WT cells (lane 4 vs. 5, Figure 6G). Ubiquitination of nephrin was only seen in cells treated with the proteasomal inhibitor MG132 (lane 2-3 vs. $4-5$, Figure $6 \mathrm{G}$ ), pointing to proteosomal involvement in this process. Moreover, in line with the in vivo data (Figure 6A), nephrin interacted strongly with $\mathrm{BiP}$ in $\mathrm{HRD1^{-/ }}$ HEK293T cells (lane 2 vs. 3, Figure 6G). Next, we determined the half-life of nephrin in transfected HEK293T cells treated with the translation inhibitor cycloheximide (CHX). Cells were pretreated with brefeldin A (BFA) to block ER exit, and, as a result, nephrin was predominantly in the $b$ form (Figure 6H). Nephrin in WT cells quickly decreased, with a half-life of 2 hours; however, that process was significantly delayed in $\mathrm{HRD1}^{-/-}$cells, with a nephrin halflife beyond 4 hours (Figure $6 \mathrm{H}$ ).

What is the significance of ERAD-mediated degradation of misfolded WT nephrin? To answer this question, we tested whether ERAD attenuates the aggregation of misfolded nephrin proteins. Indeed, the abundance of nephrin-containing high-molecular-weight (HMW) complexes with a molecular weight of more than $250 \mathrm{kDa}$ was increased by approximately 1.4 -fold in transfected $\mathrm{Hrd1}^{-/-}$cells compared with that in WT cells (Figure 6I). These HMW complexes were formed via disulfide bonds, as they were sensitive to the treatment of the reducing agent $\beta$-mercaptoethanol (Figure 6I). Taken together, we concluded that nephrin is a bona fide substrate of SEL1L-HRD1 ERAD and that ERAD of misfolded nephrin is required for its maturation in the ER.
Pathogenic nephrin mutants are SEL1L-HRD1 ERAD substrates. To further establish the pathological importance of SEL1L-HRD1 ERAD, we explored its role in the maturation of pathogenic nephrin mutants (Figure 7A). Nephrin was predicted to consist of 8 to 9 extracellular Ig domains, followed by a fibronectin (FN) type III domain, a TM region, and a cytosolic tail $(3,7)$. We first performed structural modeling analysis of nephrin using a recently developed program for contact-guided protein structure prediction, C-I-TASSER (67), which predicted that, in addition to the FN and TM domains, nephrin protein consists of 9 Ig domains (Figure 7A).

Among many nephrin mutants identified in humans with congenital nephrotic syndrome, a subset of mutants is known to cause, to a certain extent, ER retention $(15,64,65)$. We randomly chose 6 nephrin missense mutations - I171N, G270C, S366R, S724C, R743C, and L832P - located at different Ig domains - for further analyses (Figure 7A). C-I-TASSER analysis of each mutant revealed how each mutation changed the local conformation of and interaction with nearby residues (Figure 7, B and C, and Supplemental Figure 9, A-D). For example, the I171N mutation alters the formation of a hydrophobic core consisting of I171, P169, and V198, whereas the G270C mutation leads to the formation of a C270-C265 disulfide bond and abolished the C317-C265 disulfide bond (Figure 7, B and $\mathrm{C}$ ). Next, we measured the impact of each mutation on protein folding in terms of $\Delta \Delta G$, i.e., the free energy change caused by a single mutation, using EvoEF2 (68), a newly developed empirical force field for protein design and mutation effect analysis. $\Delta \Delta \mathrm{G}$ of these mutants ranged from +37.8 (S724C) to +230.9 (S366R) versus 0 (WT) (in blue, Figure 7A). A higher $\Delta \Delta \mathrm{G}$ indicates less stability, suggesting that these mutants are likely unstable.

Unlike WT nephrin, all 6 mutants appeared on SDS-PAGE predominantly (over $80 \%-100 \%$ ) as the fast-migrating $b$ form in both WT and HRD1-/- HEK293T cells (Figure 7D and quantitated in Figure 7E). Consistently, EndoH digestion revealed that these 6 nephrin mutants were EndoH sensitive, i.e., retained in the ER (Figure 7F and Supplemental Figure 9E), which was confirmed using confocal microscopy for G270C (Supplemental Figure 9F). Furthermore, all mutants were degraded by SEL1L-HRD1 ERAD, as they were stabilized in $\mathrm{HRD1}^{-/}$HEK293T cells treated with $\mathrm{CHX}$ for 4 hours (Figure $7 \mathrm{G}$ and quantitated in Figure $7 \mathrm{H}$ ). Hence, these data showed that all 6 human mutants were retained in the ER, where they were degraded by SEL1L-HRD1 ERAD.

ERAD of mutant nephrin reduces its aggregation propensity and pathogenicity. Last, we explored the pathological significance of ERAD-mediated degradation of human mutant nephrin. ERAD deficiency triggered most nephrin mutants (with the exception of S366R) to form HMW complexes (Figure 8, A and B), and the effect was much more dramatic than that seen in WT nephrin (vs. lanes 1 and 2, Figure 8A). These mutants also formed more detergent-insoluble (0.5\% NP40-insoluble) protein aggregates in ERAD-deficient cells (Figure 8C). Therefore, these data show that SEL1L-HRD1 ERAD mediated the degradation of nephrin mutants, thereby attenuating their aggregation.

Although these mutants are aggregation prone, 1 allele is insufficient to cause disease (i.e., autosomal-recessive). Hence, we asked whether ERAD attenuates the pathogenic effect of mutants toward the WT allele. WT nephrin was tagged with Myc, while the mutants (I171N and G270C) were either untagged or tagged 
with Flag to thereby distinguish the 2 forms. In WT HEK293T cells, coexpression of mutant nephrin had little to no effect $(10 \%-$ $20 \%$ increase) on the HMW formation of WT nephrin compared with WT nephrin alone (lanes 5 and 6 vs. 2, Figure 8D). However, in $H R D 1^{-/}$cells, coexpression of mutant nephrin significantly enhanced by 2.5- to 3-fold the HMW complex formation of WT nephrin compared with WT nephrin alone (lanes 10 and 11 vs. 7, Figure 8D). Moreover, coexpression of mutant nephrin reduced the total WT nephrin protein levels in WT cells in a dose-dependent manner, but had no effect on the maturation efficiency as quantitated by the percentage of nephrin $a$ in total nephrin (lane 2 vs. lanes 3 and 4 and 5 and 6, Figure 8E, and quantitated in Figure $8 \mathrm{~F})$. By contrast, coexpression of mutant nephrin in $\mathrm{HRD1^{-/ }}$ cells did not affect total WT nephrin levels, but reduced the percentage of the nephrin $a$ form in a dose-dependent manner (lane 8 vs. lanes 9 and 10 and 11 and 12, Figure 8E, and quantitated in Figure $8 \mathrm{~F}$ ). These data suggested that, although mutant nephrin promotes the degradation of WT nephrin in ERAD-competent cells, it only interferes with the maturation of WT nephrin in ERAD-deficient cells, not in ERAD-competent cells. Thus, we concluded that SEL1L-HRD1 ERAD degrades nephrin mutants, thereby ensuring normal maturation of the WT allele.

\section{Discussion}

Our data demonstrate that SEL1L-HRD1 ERAD in podocytes plays a critical role in the formation of the slit diaphragm and glomerular filtration function. SEL1L deficiency in podocytes impairs slit diaphragm integrity and leads to podocytopathy, congenital nephrotic syndrome, and renal failure, starting at 3 to 5 weeks after birth. Our side-by-side comparison with mice carrying podocyte-specific deletion of the UPR sensor IRE1 $\alpha$ showed that IRE1 $\alpha$ was dispensable for physiology at the same age, in keeping with the conclusions from previous studies $(24,25)$. Together with recent studies $(27,28,34,69)$, these findings confirm the vital importance of SEL1L-HRD1 ERAD in physiology.

Our data further suggest that the effect of ERAD in podocytes is largely substrate dependent. Nascent nephrin protein is misfolding prone and ubiquitinated by SEL1L-HRD1 ERAD for proteasomal degradation. In the absence of ERAD, misfolded nephrin accumulates in the ER and may undergo further refolding or interfere with the normal maturation process for nascent nephrin protein. Indeed, our data showed that interaction between nephrin and BiP was enhanced in the absence of ERAD, as were the nephrin-containing HMW protein complexes. Moreover, the proportion of the ER-retained nephrin $b$ form was progressively increased in Sel1L ${ }^{\text {PodCre }}$ kidneys starting at 3 weeks of age, in direct contrast to WT kidneys, where the mature nephrin $a$ form was elevated with age. Hence, we conclude that SEL1L-HRD1 ERAD plays an indispensable role in podocyte function, at least in part, by regulating the maturation of nascent nephrin protein in the ER. Although it has been reported that intracellular distribution of nephrin is altered in humans with FSGS $(70,71)$, it remains unclear whether SEL1L-HRD1 ERAD plays a role in the disease pathogenesis. Our initial data showed that expression of SEL1L-HRD1 ERAD was altered in patients with FSGS; however, a significantly larger sample size, with consideration of patient age, sex, race, disease state, medications, and the nature of the control samples, is required to address this question in the future.
While some nephrin mutations in humans with congenital nephrotic syndrome - mostly autosomal-recessive - are known to be retained in the ER $(14,15)$, our understanding of the molecular events associated with the biogenesis of nascent nephrin proteins, especially in regard to their interactions with quality control machineries, remained largely unexplored. In this study, we report that SEL1L-HRD1 ERAD mediates the degradation of all 6 pathogenic mutants, which may attenuate not only their accumulation and self-aggregation in the ER, but also their pathogenicity toward the WT allele. In the absence of ERAD, these nephrin mutants readily form HMW and insoluble aggregates, which include some WT nephrin protein. Indeed, the role of SEL1L-HRD1 ERAD in podocytes bears resemblance to its role in the maturation of the prohormones AVP and POMC in the pathogenesis of diabetes insipidus and obesity, respectively $(37,38)$. Hence, we speculate that enhancement of SEL1LHRD1 ERAD activity may reduce the dominant negative effects of mutant alleles in human diseases. How SEL1L-HRD1 ERAD identifies and targets misfolded nephrin remains unclear.

Cells constantly live under various physiological and pathological stresses. This is especially true for podocytes, which are responsible for maintaining the filtration barrier and face not only mechanical but also cellular stresses (including ER and oxidative stresses) and immunological challenges. These cells, therefore, have a high capacity to preserve function by synthesizing GBM membrane components, forming the slit diaphragm, and enhancing endothelial cell survival (1). ER homeostasis is maintained mainly by 3 principal quality control machineries, namely, ERAD, the UPR, and autophagy. The observation that deletion of Ire $1 a-X b p 1$ or Atg5 has minimal effect on podocyte function (refs. 24-26 and this study) again supports the notion that podocytes are highly resilient and adaptive. Moreover, our findings highlight the fundamental importance of SEL1L-HRD1 ERAD as the first line of defense against misfolded proteins in the ER, and in the case of podocytes, we found that SEL1L-HRD1 ERAD was essential for slit diaphragm formation. It is worth pointing out that, in 7- to 10-week-old mice, we observed podocyte loss, as revealed by SEM analysis of glomerulus and the reduction in total levels of nephrin and synaptopodin proteins. These finding suggested that podocytes may not be able to tolerate chronic SEL1L-HRD1 ERAD deficiency. However, as disease was already initiated at 3 to 5 weeks of age in $\operatorname{Sel1} L^{\text {PodCre }}$ mice, in which glomeruli and podocytes appeared largely normal, we believe that podocyte SEL1L-HRD1 ERAD controlled disease initiation, not as a result of cell death, but rather in a substrate-specific manner.

This study focused on the maturation of nephrin because of its pathophysiological importance and, more important, because of the similarity between the Sel1L-and nephrin-deficient mouse models. However, since nephrin-deficient mice die within 24 hours after birth (8) versus approximately 13 weeks for podocyte-specific Sel1L-deficient mice, we acknowledge that SEL1L-HRD1 ERAD deficiency probably does not cause a complete loss of function of nephrin. This is consistent with the biochemical results showing that some nephrin still matured beyond the ER, even at 5 to 7 weeks of age. In addition, we speculate that ERAD in podocytes may regulate the maturation of other proteins in a manner similar to that of nephrin, proteins such as those involved in the generation of 
secondary and tertiary FPs, which may also contribute to the early lethality of Sel1L $L^{\text {PodCre }}$ mice. While all these possibilities require further investigation, our findings demonstrate a crucial role of SEL1L-HRD1 ERAD in podocyte function in health and disease.

\section{Methods}

\section{Human studies}

Human tissue samples and immunofluorescence staining. Deidentified formalin-fixed, paraffin-embedded kidney sections $(3 \mu \mathrm{m})$ from patients who were found to have FSGS on kidney biopsy (FSGS1, -2, -3) and from living kidney donors were obtained from the Department of Pathology, University of Michigan Medical School, Ann Arbor, Michigan, USA, and used for SEL1L and HRD1 immunostaining. Antigen retrieval was performed in boiling $10 \mathrm{mM}$ sodium citrate buffer $\mathrm{pH}$ 6.0) for 15 minutes. Antibodies were diluted in 5\% donkey serum and $0.3 \%$ Triton X-100 in PBS. Slides were placed in a humidified chamber at $4^{\circ} \mathrm{C}$ overnight with primary antibodies. The next day, slides were incubated with secondary antibodies for 1 hour at room temperature. After 3 washes with $0.1 \%$ Tween in TBS (TBST), slides were incubated with anti-WT1 Alexa Fluor 647 for 1 hour at room temperature. Following washes with TBST and distilled water, $10 \mu \mathrm{L}$ ProLong Gold Antifade mounting media with DAPI (Invitrogen, Thermo Fisher Scientific, P36931) was applied. Samples were imaged with identical parameters under a Nikon A1 Confocal Microscope at the University of Michigan Imaging Core.

Antibodies. The following antibodies were used for immunofluorescence of human samples: SEL1L (43) (home-made E12049, specific for both human and mouse, rabbit, 1:500); HRD1 (provided by Richard Wojcikiewicz, SUNY Upstate Medical University, Syracuse, New York, USA; rabbit, 1:50 for immunostaining); and WT1-Alexa Fluor 647 (Abcam, ab202639, 1:500).

scRNA-Seq of the human kidney. Human kidney tissue obtained from the unaffected, distant tumor site of a 43-year-old male patient undergoing nephrectomy (warm and cold ischemic time $<5$ minutes). Experimental details are described in the Supplemental Methods. scRNA-seq data were deposited in the NCBI's BioProject database (accession number PRJNA700694; https://www.ncbi.nlm.nih.gov/ bioproject/700694).

\section{Mouse studies}

Mice. Sel1L $L^{f l / f l}$ mice (35) were crossed with mice expressing the Cre transgene driven by the podocin promoter (56) on a C57BL/6 background to generate podocyte-specific Sel1L-deficient (Sel1L $\left.{ }^{\text {PodCre }}\right)$ mice. Their littermates, Sel1L $L^{f / f l}(\mathrm{WT})$ and heterozygous (Sel1L $\left.{ }^{\text {Podcre/+}}\right)$ mice were included as control cohorts. Ire $1 a^{f / f l}$ mice (72) were used to generate Ire $1 a^{\text {PodCre }}$ mice, applying the same breeding scheme. All mice were housed in an ambient-temperature room under a 12-hour light cycle and fed a normal chow diet (13\% fat, $57 \%$ carbohydrate, and $30 \%$ protein, LabDiet 5LOD). Weekly measurements of body weight were performed at the same time of day. Both male and female mice at the ages of $3,5,7$, and 10 weeks were used in the studies.

Tissue fixation. After the mice were anesthetized, the kidneys were perfused with cold PBS for 2 minutes and then with cold fixation buffer A (4\% paraformaldehyde [50-980-487], and 2.5\% glutaraldehyde [16000], in Sorensen's buffer [11600-05]; all from Electron Microscopy Sciences) for electron microscopy (EM) and H\&E staining, or with buffer B (10\% formalin in PBS) for immunofluorescence staining. The samples were sent to the Research Histology and Immunohistochemistry Core at the University of Michigan Medical School for paraffin processing, embedment, and H\&E staining.

Immunofluorescence staining and confocal microscopy. The kidneys were fixed in fixation buffer (10\% formalin in PBS) at $4^{\circ} \mathrm{C}$ overnight, transferred to cold PBS, and incubated at $4^{\circ} \mathrm{C}$ overnight, and then transferred to cold PBS with $20 \%$ sucrose at $4^{\circ} \mathrm{C}$ for another overnight incubation. Next, the samples were frozen in Tissue-Tek O.C.T. Compound (Electron Microscopy Sciences) and kept at $-80^{\circ} \mathrm{C}$. The frozen sections were prepared by cutting into $5 \mu \mathrm{m}$ sections with a cryostat (Leica, CM1950). WT and knockout tissues from the same litter were prepared on the same slide under the same conditions and kept at $-80^{\circ} \mathrm{C}$. For staining, the slides were washed 3 times in PBS at room temperature followed by blocking buffer ( $5 \%$ BSA, $0.1 \%$ Tween, TBS) for 30 minutes at room temperature. Primary antibodies were diluted in the blocking buffer and applied to the tissue areas followed by incubation at $4^{\circ} \mathrm{C}$ overnight. The next morning, the slides were washed 3 times for 10 minutes with TBST, and then a secondary antibody solution was applied and incubated for 2 hours at room temperature. Samples were washed 3 times for 10 minutes with TBST and briefly washed with water before mounting (ProLong Gold Antifade Reagent with DAPI, Invitrogen, Thermo Fisher Scientific). Tissues on the same slide were imaged using identical imaging parameters with a Nikon A1 Confocal Laser Microscope (Microscopy Core at the University of Michigan). Signal intensities as well as the distribution patterns were compared under the same conditions.

Antibodies. HRD1, SEL1L, and WT1 antibodies were used for immunostaining as described above. The following antibodies were used: SEL1L (Abcam, ab78298; 1:1000 for Western blotting); podocin (MilliporeSigma, P0372; 1:100 for immunostaining and ABclonal, A17337; 1:3000 for Western blotting); HRD1 (Proteintech, 13473-1AP; 1:3000 for Western blotting); synaptopodin (Santa Cruz Biotechnology, sc-515842; 1:100 for immunostaining and ABclonal, A12049; 1:2000 for Western blotting); nephrin (ABclonal, A3048; 1:2000 for Western blotting, 1:100 for immunostaining); KDEL (Abcam, ab12223; 1:200 for immunostaining); ZO1 (Thermo Fisher Scientific, 33-9100; 1:100 for immunostaining); BiP (Abcam, ab21685; 1:5000 for Western blotting, 1:200 for immunostaining); CD2AP (Proteintech, 51046-1-AP; 1:300 for immunostaining); ubiquitin (Ub) (Santa Cruz Biotechnology, sc-8017; 1:1000 for Western blotting); Myc (MilliporeSigma, C3956; 1:5000 for Western blotting); Flag (MilliporeSigma, F-1804; 1:5000 for Western blotting); HSP90 (Santa Cruz Biotechnology, sc-13119; 1:5000 for Western blotting); and histone H2A (Cell Signaling Technology, 2578; 1:2000 for Western blotting). The following secondary antibodies were used for Western blotting: goat anti-rabbit IgG HRP (Bio-Rad, 5196-2504; 1:5000) and anti-mouse IgG HRP (Bio-Rad, 1706516; 1:5000). The following secondary antibodies were used for immunostaining: anti-mouse IgG Alexa Fluor 594 (115-585-044); anti-mouse IgG Alexa Fluor 488 (715-545-150); anti-rabbit IgG Alexa Fluor 594 (111-585-144); and anti-rabbit IgG Alexa Fluor 488 (711-545-152) (all from Jackson ImmunoResearch Laboratories and used at 1:500-1:1000).

Imaging data analysis. SEL1L/HRD1 signal intensity per podocyte was measured using ImageJ software (NIH) and was further analyzed using ggplot2 as described in the Human studies section. ImageJ was used to quantitate the glomerulus size and the index of mesangial 
hyperplasia. To determine the index of mesangial hyperplasia, the $\mathrm{H} \& \mathrm{E}$ stain-positive area measured in each glomerulus was divided by the area of whole glomerulus.

TEM and advanced SEM. EM samples were prepared using previously described standard methods $(19,58)$. The samples were submitted to the University of Michigan Microscope and Image Analysis core facility for washing and embedment according to standard procedures. Embedded samples were analyzed using the JEOL USA JEM1400 Plus Electron Microscope for TEM and the AMRAY 1910 Field Emission Scanning Electron Microscope for SEM. For ultra-high-resolution advanced SEM, samples were examined under the Thermo Fisher Helios 650 Nanolab SEM at the Michigan Center for Materials Characterization.

\section{In vitro studies}

Cell lines. HEK293T and N2a cells, obtained from the American Type Culture Collection (ATCC), were cultured in DMEM (Corning, $10017 \mathrm{CV}$ ) containing $10 \% \mathrm{FBS}$ and $1 \%$ penicillin-streptomycin. $\mathrm{HRD1}^{-/-} \mathrm{HEK} 293 \mathrm{~T}$ and N2a cells were generated as described previously $(38,49)$. To generate $H R D 1^{-/-}$podocytes, immortalized CIHP-1 human podocytes $(73)$ were grown at $33^{\circ} \mathrm{C}$ to $80 \%$ confluence in RPMI 1640 (Corning, 10-040-CM) supplemented with insulin-transferrin-selenium (Gibco, Thermo Fisher Scientific, 51500-056), 10\% FBS, and $1 \%$ penicillin-streptomycin, followed by subculturing at $1: 3$, and then incubation at $37^{\circ} \mathrm{C}$ for 14 days to induce differentiation. On day 10 , the cells were transduced with the lentiviral CRISPR system targeting HRD1 as previously described (49). The guide sequences for HRD1 were as follows: GGGACAAAGGCCTGGATGTAC (guide 1) and GGGCCAGCCTGGCGCTGACCG (guide 2).

Plasmids. HA-Ub, HRD1-WT-Myc, and HRD1-C2A-Myc plasmids were described previously (49). Mouse nephrin and Myc-nephrin plasmids, from which Flag-tagged nephrin was generated by PCR, were previously described. Quick-change mutagenesis was performed to generate nephrin point mutants using the mouse nephrin constructs (49). The following primers were used for mutagenesis: I171N (5'-AAGCCAGCACCTGACAACATCTTCATCCAGGGT-3', 5'-ACCCTGGATGAAGATGTTGTCAGGTGCTGGCTT-3'); G270C (5'-TGCATAGCCAGAGGTtGTAATCCACCTGCGACC-3', 5'-GGTCGCAGGTGGATTACAACCTCTGGCTATGCA-3'); S366R (5' -TGCCTTACCAAGTCCAGACGCCCACGGGTCCTG-3', 5'-CAGGACCCGTGGGCGTCTGGACTTGGTAAGCA-3'); S724C (5' -CTACACTGCCAGAACTGCGAGGGCACCGCCGAG-3', $5^{\prime}$ - CTCGGCGGTGCCCTCGCAGTTCTGGCAGTGTAG-3'); R743C (5'-TATGCTCCCACCATCTGTGCCCTGAAGGACCCT-3', 5'-AGGGTCCTTCAGGGCACAGATGGTGGGAGCATA-3'); and L832P (5'-AGAGGACTGGTTCGTCCTGTTGTCCGATTTGCC-3', 5'-GGCAAATCGGACAACAGGACGAACCAGTCCTCT-3').

In vitro drug treatment. The experiments involving treatments with CHX, MG132, and BFA were performed as described previously $(37,41,48,49)$. Briefly, transfected HEK293T cells were pretreated with BFA $(1 \mu \mathrm{g} / \mathrm{mL})$ for 30 minutes, followed by CHX for 2 and 4 hours. MG132 $(10 \mu \mathrm{M})$ was added to the culture 5 hours before the experiments were performed.

${ }^{35} \mathrm{~S}$ pulse-chase experiment. HEK293T cells were transfected with Myc- or Flag-nephrin constructs followed by a pulse-chase experiment. HEK293T cells were pulse-labeled with $100 \mu \mathrm{Ci} / \mathrm{mL}\left[{ }^{35} \mathrm{~S}\right]$-cysteine and methionine at $37^{\circ} \mathrm{C}$ for 30 minutes. Following a 30-minute pulse, cells were cultured in chase medium (regular media supplemented with $10 \mathrm{mM}$ HEPES, $5 \mathrm{mM}$ cysteine, $5 \mathrm{mM}$ methionine) for the indicated durations. Cells were then washed with ice-cold HBSS buffer (Gibco, Life Technologies, Thermo Fisher Scientific), snap-frozen in liquid nitrogen, and lysed in NP-40 lysis buffer (1\% NP-40, $150 \mathrm{mM} \mathrm{NaCl}, 1 \mathrm{mM}$ EDTA, $50 \mathrm{mM}$ Tris- $\mathrm{HCl} \mathrm{pH}$ 8.0) supplemented with $100 \mathrm{X}$ proteinase inhibitor cocktail (MilliporeSigma), $1 \mathrm{mM}$ PMSF (MilliporeSigma), and $10 \mathrm{mM} \mathrm{N}$-ethylmaleimide. Lysates were used in the immunoprecipitation as described above. Denaturing samples were prepared by adding $2 \times$ denaturing sample buffer into the immunoprecipitated beads followed by 10 minutes of boiling at $95^{\circ} \mathrm{C}$.

\section{Statistics}

All experiments were conducted at least twice or with at least 2 biological replicates. Results are expressed as the mean \pm SEM. Unless otherwise specified, comparisons between 2 groups and between multiple groups were made using an unpaired, 2-tailed Student's $t$ test and 1-way ANOVA followed by Tukey's multiple-comparison test, respectively. For survival analysis, the Kaplan-Meier method and the log-rank test were used. $P$ values of less than 0.05 were considered statistically significant.

\section{Study approval}

The human study was approved by the IRB of the University of Michigan (HUM00002468). All animal procedures were approved by and conducted in accordance with the IACUC of the University of Michigan Medical School (PRO00008989).

\section{Author contributions}

SY initiated, designed, and performed in vivo studies. XW designed and performed some in vitro experiments and performed structural analysis. GZ repeated many in vivo and in vitro experiments and performed some of the in vitro experiments. CLO, RM, EO, $\mathrm{JBH}$, and MB provided scRNA-Seq data and human kidney samples. MT assisted with and performed EM studies. LL, ZZ, XX, and YX performed some of the biochemical assays. YZ helped with structural analysis. WZ and RH performed imaging quantitation of human samples. CHAT, CCAH, RV, PG, HM, SS, MB, and ML provided critical insights and discussions. LQ conceived the study, designed experiments, and wrote the manuscript. All authors commented on and approved the manuscript. Both scientific and intellectual contributions were taken into account in deciding the order of the co-first authors.

\section{Acknowledgments}

We would like to thank Richard Wojcikiewicz for the HRD1 antibody. We thank Chengxin Zhang for technical assistance in depositing sequencing data. We thank members of the Arvan and Qi laboratories for their technical assistance and insightful discussions. We also thank the University of Michigan Comprehensive Cancer Center (UMCCC) Tissue Core, Advanced Genomics Core, Imaging Laboratory of Michigan Diabetes Research Center, the Microscopy Core of Biomedical Research Core Facilities, and the Michigan Center for Materials Characterization for their support. This work is supported by the NIH (1R01CA163910, to CCAH and 1R35GM136422, to YZ); the National Natural Science Foundation of China (81620108004 and 81830025, to ML); the National Center for Advancing Trans- 
lational Sciences (NCATS), NIH (UL1TR002240, to MB); and the NIH (R01DK120330, 1R01DK120047, 1R35GM130292, and a pilot grant from P30DK081943, to LQ). SY is supported in part by a Frontiers Science Center for Cell Responses grant from Nankai University (C029205001). ZZ is supported by an American Diabetes Association (ADA) Postdoctoral Fellowship (1-19-PDF-093).
Address correspondence to: Ling Qi, University of Michigan Medical School, 5325 Brehm Tower, 1000 Wall St., Ann Arbor, Michigan 48105, USA. Phone: 734.936.4720; Email: lingq@med.umich. edu. Or to: Sei Yoshida, Nankai University College of Life Sciences, 94 Weijin Road, Tianjin, China 300071. Phone: 180.2000.3587; Email: seiyoshi@nankai.edu.cn.
1. Garg P. A review of podocyte biology. Am J Nephrol. 2018;47(suppl 1):3-13.

2. Shih NY, et al. Congenital nephrotic syndrome in mice lacking CD2-associated protein. Science. 1999;286(5438):312-315.

3. Kestila M, et al. Positionally cloned gene for a novel glomerular protein-nephrin-is mutated in congenital nephrotic syndrome. Mol Cell. 1998;1(4):575-582.

4. Boute N, et al. NPHS2, encoding the glomerular protein podocin, is mutated in autosomal recessive steroid-resistant nephrotic syndrome. Nat Genet. 2000;24(4):349-354.

5. Mollet $\mathrm{G}$, et al. Podocin inactivation in mature kidneys causes focal segmental glomerulosclerosis and nephrotic syndrome. J Am Soc Nephrol. 2009;20(10):2181-2189.

6. Chen YM, Liapis H. Focal segmental glomerulosclerosis: molecular genetics and targeted therapies. BMC Nephrol. 2015;16:101.

7. Lenkkeri U, et al. Structure of the gene for congenital nephrotic syndrome of the finnish type (NPHS1) and characterization of mutations. $A m J$ Hum Genet. 1999;64(1):51-61.

8. Putaala $\mathrm{H}$, et al. The murine nephrin gene is specifically expressed in kidney, brain and pancreas: inactivation of the gene leads to massive proteinuria and neonatal death. Hum Mol Genet. 2001;10(1):1-8.

9. Holmberg C, et al. Management of congenital nephrotic syndrome of the Finnish type. Pediatr Nephrol. 1995;9(1):87-93.

10. Holmberg C, et al. Renal transplantation in small children with congenital nephrotic syndrome of the Finnish type. Transplant Proc. 1991;23(1 pt 2):1378-1379.

11. Park SJ, et al. Discovery of endoplasmic reticulum calcium stabilizers to rescue ER-stressed podocytes in nephrotic syndrome. Proc Natl Acad Sci U S A. 2019;116(28):14154-14163.

12. Jalanko H. Congenital nephrotic syndrome. Pediatr Nephrol. 2009;24(11):2121-2128.

13. Rantanen M, et al. Nephrin TRAP mice lack slit diaphragms and show fibrotic glomeruli and cystic tubular lesions. J Am Soc Nephrol. 2002;13(6):1586-1594.

14. Martin CE, Jones N. Nephrin signaling in the podocyte: an updated view of signal regulation at the slit diaphragm and beyond. Front Endocrinol (Lausanne). 2018;9:302.

15. Liu L, et al. Defective nephrin trafficking caused by missense mutations in the NPHS1 gene: insight into the mechanisms of congenital nephrotic syndrome. Hum Mol Genet. 2001;10(23):2637-2644.

16. Cybulsky AV. The intersecting roles of endoplasmic reticulum stress, ubiquitin-proteasome system, and autophagy in the pathogenesis of proteinuric kidney disease. Kidney Int.
2013;84(1):25-33.

17. Cybulsky AV. Endoplasmic reticulum stress, the unfolded protein response and autophagy in kidney diseases. Nat Rev Nephrol. 2017;13(11):681-696.

18. Inagi $R$, et al. Proteostasis in endoplasmic reticulum - new mechanisms in kidney disease. Nat Rev Nephrol. 2014;10(7):369-378.

19. Inoki K, et al. mTORC1 activation in podocytes is a critical step in the development of diabetic nephropathy in mice. J Clin Invest. 2011;121(6):2181-2196.

20. Ito $\mathrm{N}$, et al. mTORC1 activation triggers the unfolded protein response in podocytes and leads to nephrotic syndrome. Lab Invest. 2011;91(11):1584-1595.

21. Chen YM, et al. Laminin $\beta 2$ gene missense mutation produces endoplasmic reticulum stress in podocytes. JAm Soc Nephrol. 2013;24(8):1223-1233.

22. Cybulsky AV, et al. Glomerular epithelial cell injury associated with mutant alpha-actinin-4. Am J Physiol Renal Physiol. 2009;297(4):F987-F995.

23. Pieri $\mathrm{M}$, et al. Evidence for activation of the unfolded protein response in collagen IV nephropathies. JAm Soc Nephrol. 2014;25(2):260-275.

24. Kaufman DR, et al. Deletion of inositol-requiring enzyme-1 $\alpha$ in podocytes disrupts glomerular capillary integrity and autophagy. Mol Biol Cell. 2017;28(12):1636-1651.

25. Hassan $\mathrm{H}$, et al. Essential role of $\mathrm{X}$-box binding protein-1 during endoplasmic reticulum stress in podocytes. J Am Soc Nephrol. 2016;27(4):1055-1065

26. Hartleben B, et al. Autophagy influences glomerular disease susceptibility and maintains podocyte homeostasis in aging mice. J Clin Invest. 2010;120(4):1084-1096.

27. Bhattacharya A, Qi L. ER-associated degradation in health and disease - from substrate to organism. J Cell Sci. 2019;132(23):jcs232850.

28. Hwang J, Qi L. Quality control in the endoplasmic reticulum: crosstalk between ERAD and UPR pathways. Trends Biochem Sci. 2018;43(8):593-605.

29. Guerriero CJ, Brodsky JL. The delicate balance between secreted protein folding and endoplasmic reticulum-associated degradation in human physiology. Physiol Rev. 2012;92(2):537-576.

30. Mueller B, et al. SEL1L, the homologue of yeast Hrd3p, is involved in protein dislocation from the mammalian ER. J Cell Biol. 2006;175(2):261-270.

31. Mueller B, et al. SEL1L nucleates a protein complex required for dislocation of misfolded glycoproteins. Proc Natl Acad Sci U S A. 2008;105(34):12325-12330.

32. Hampton RY, et al. Role of $26 \mathrm{~S}$ proteasome and HRD genes in the degradation of 3-hydroxy-3-methylglutaryl-CoA reductase, an integral endoplasmic reticulum membrane protein. Mol Biol Cell.1996;7(12):2029-2044.

33. Plemper RK, et al. Genetic interactions of Hrd3p and Der3p/Hrd1p with Sec61p suggest a retro-translocation complex mediating protein transport for ER degradation. J Cell Sci. 1999;112(Pt 22):4123-4134.

34. Qi L, et al. New insights into the physiological role of endoplasmic reticulum-associated degradation. Trends Cell Biol. 2017;27(6):430-440.

35. Sun S, et al. Sel1L is indispensable for mammalian endoplasmic reticulum-associated degradation, endoplasmic reticulum homeostasis, and survival. Proc Natl Acad Sci U S A. 2014;111(5):E582-E591.

36. Fujita $\mathrm{H}$, et al. The E3 ligase synoviolin controls body weight and mitochondrial biogenesis through negative regulation of PGC-1 1 . EMBO J. 2015;34(8):1042-1055.

37. Shi G, et al. ER-associated degradation is required for vasopressin prohormone processing and systemic water homeostasis. JClin Invest. 2017;127(10):3897-3912.

38. Kim GH, et al. Hypothalamic ER-associated degradation regulates POMC maturation, feeding, and age-associated obesity.J Jlin Invest. 2018;128(3):1125-1140.

39. Ji Y, et al. The Sel1L-Hrd1 endoplasmic reticulum-associated degradation complex manages a key checkpoint in B cell development. Cell Rep. 2016;16(10):2630-2640.

40. Sun S, et al. Epithelial Sel1L is required for the maintenance of intestinal homeostasis. Mol Biol Cell. 2016;27(3):483-490.

41. Bhattacharya A, et al. Hepatic Sel1L-Hrd1 ER-associated degradation (ERAD) manages FGF21 levels and systemic metabolism via CREBH. EMBO J. 2018;37(22):e99277.

42. Shrestha N, et al. Sel1L-Hrd1 ER-associated degradation maintains $\beta$ cell identity via TGF $\beta$ signaling. J Clin Invest. 2020;130(7):3499-3510.

43. Zhou Z, et al. Endoplasmic reticulum-associated degradation regulates mitochondrial dynamics in brown adipocytes. Science. 2020;368(6486):54-60.

44. Wu T, et al. Hrd1 suppresses Nrf2-mediated cellular protection during liver cirrhosis. Genes Dev. 2014;28(7):708-722.

45. Xu Y, et al. The ER membrane-anchored ubiquitin ligase $\mathrm{Hrd} 1$ is a positive regulator of T-cell immunity. Nat Commun. 2016;7:12073.

46. Wei J, et al. HRD1-ERAD controls production of the hepatokine FGF21 through CREBH polyubiquitination. $Е M B O$ J. 2018;37(22):e98942.

47. Hu Y, et al. Endoplasmic reticulum-associated degradation (ERAD) has a critical role in supporting glucose-stimulated insulin secretion in pancreatic $\beta$-cells. Diabetes. 2019;68(4):733-746.

48. Sha H, et al. The ER-associated degradation adaptor protein Sel1 $\mathrm{L}$ regulates LPL secretion and lipid metabolism. Cell Metab. 2014;20(3):458-470.

49. Sun S, et al. IRE1a is an endogenous substrate of endoplasmic-reticulum-associated degradation. Nat Cell Biol. 2015;17(12):1546-1555. 
50. Kato M, et al. An endoplasmic reticulum stress-regulated lncRNA hosting a microRNA megacluster induces early features of diabetic nephropathy. Nat Commun. 2016;7:12864.

51. Hodgin JB, et al. Glomerular aging and focal global glomerulosclerosis: a podometric perspective. JAm Soc Nephrol. 2015;26(12):3162-3178.

52. Menon R, et al. Single-cell analysis of progenitor cell dynamics and lineage specification in the human fetal kidney. Development. 2018;145(16):dev164038.

53. Vashistha $\mathrm{N}$, et al. Direct and essential function for Hrd3 in ER-associated degradation. Proc Natl Acad Sci U S A. 2016;113(21):5934-5939.

54. Gardner RG, et al. Endoplasmic reticulum degradation requires lumen to cytosol signaling. Transmembrane control of Hrd1p by Hrd3p. JCell Biol. 2000;151(1):69-82.

55. D’Agati VD, Kaskel FJ, Falk RJ. Focal segmental glomerulosclerosis. $N$ Engl JMed. 2011;365(25):2398-411.

56. Moeller MJ, et al. Podocyte-specific expression of cre recombinase in transgenic mice. Genesis. 2003;35(1):39-42.

57. Grahammer F, et al. A flexible, multilayered protein scaffold maintains the slit in between glomerular podocytes. JCI Insight. 2016;1(9):e86177.

58 . Verma R, et al. Nephrin is necessary for podocyte recovery following injury in an adult mature glomerulus. PLoS One. 2018;13(6):e0198013.
59. Ruotsalainen V, et al. Nephrin is specifically located at the slit diaphragm of glomerular podocytes. Proc Natl Acad Sci U S A. 1999;96(14):7962-7967.

60. Rodewald R, Karnovsky MJ. Porous substructure of the glomerular slit diaphragm in the rat and mouse. J Cell Biol. 1974;60(2):423-433.

61. Wartiovaara J, et al. Nephrin strands contribute to a porous slit diaphragm scaffold as revealed by electron tomography. JClin Invest. 2004;114(10):1475-1483.

62. Kurihara H, et al. The altered glomerular filtration slits seen in puromycin aminonucleoside nephrosis and protamine sulfate-treated rats contain the tight junction protein ZO-1. Am J Pathol. 1992;141(4):805-816.

63. Macconi D, et al. Effect of angiotensin-converting enzyme inhibition on glomerular basement membrane permeability and distribution of zonula occludens-1 in MWF rats. J Am Soc Nephrol. 2000;11(3):477-489.

64. Drozdova T, et al. Nephrin missense mutations: induction of endoplasmic reticulum stress and cell surface rescue by reduction in chaperone interactions. Physiol Rep. 2013;1(4):e00086.

65. Tanigawa S, et al. Organoids from nephrotic disease-derived iPSCs identify impaired nephrin localization and slit diaphragm formation in kidney podocytes. Stem Cell Reports. 2018;11(3):727-740.

66. Yan K, et al. N-linked glycosylation is critical for the plasma membrane localization of nephrin. JAm Soc Nephrol. 2002;13(5):1385-1389.

67. Zheng W, et al. Deep-learning contact-map guided protein structure prediction in CASP13. Proteins. 2019;87(12):1149-1164.

68. Huang X, et al. EvoEF2: accurate and fast energy function for computational protein design. Bioinformatics. 2019;36(4):1135-1142.

69. Shrestha N, et al. Endoplasmic reticulum protein quality control in $\beta$ cells. Semin Cell Dev Biol. 2020;103:59-67.

70. Doublier S, et al. Nephrin redistribution on podocytes is a potential mechanism for proteinuria in patients with primary acquired nephrotic syndrome. Am J Pathol. 2001;158(5):1723-1731.

71. Doublier S, et al. Nephrin expression is reduced in human diabetic nephropathy: evidence for a distinct role for glycated albumin and angiotensin II. Diabetes. 2003;52(4):1023-1030.

72. Iwawaki T, et al. Function of IRE1 alpha in the placenta is essential for placental development and embryonic viability. Proc Natl Acad Sci US A. 2009;106(39):16657-16662.

73. Saleem MA, et al. A conditionally immortalized human podocyte cell line demonstrating nephrin and podocin expression. JAm Soc Nephrol. 2002;13(3):630-638.

74. Verma R, et al. Integrin ligation results in nephrin tyrosine phosphorylation in vitro. PLoS One. 2016;11(2):e0148906. 